International Journal of Modern Physics A

(C) World Scientific Publishing Company

\title{
IMPACT PARAMETER SPACE INTERPRETATION FOR GENERALIZED PARTON DISTRIBUTIONS
}

\author{
MATTHIAS BURKARDT* \\ Department of Physics, New Mexico State University \\ Las Cruces, New Mexico 88011, U.S.A. ${ }^{\dagger}$ \\ Received (Day Month Year) \\ Revised (Day Month Year)
}

\begin{abstract}
The Fourier transform of generalized parton distribution functions at $\xi=0$ describes the distribution of partons in the transverse plane. The physical significance of these impact parameter dependent parton distribution functions is discussed. In particular, it is shown that they satisfy positivity constraints which justify their physical interpretation as a probability density. The generalized parton distribution $H$ is related to the impact parameter distribution of unpolarized quarks for an unpolarized nucleon, $\tilde{H}$ is related to the distribution of longitudinally polarized quarks in a longitudinally polarized nucleon, and $E$ is related to the distortion of the unpolarized quark distribution in the transverse plane when the nucleon has transverse polarization. The magnitude of the resulting transverse flavor dipole moment can be related to the anomalous magnetic moment for that flavor in a model independent way.

Keywords: Generalized Parton Distributions; Form Factors
\end{abstract}

\section{Introduction}

Deep-inelastic scattering (DIS) experiments have not only played a crucial role in establishing QCD as a theory of strong interactions but have also been an important tool for exploring the quark-gluon structure of hadrons. In the Bjorken scaling limit these experiments allow probing parton distribution functions (PDFs), which have a very physical interpretation as the (probability) density for finding partons carrying the fraction $x$ of the target's total momentum in the infinite momentum (or light-cone) frame. In particular, due to the probabilistic interpretation of PDFs, results from DIS experiments have contributed substantially to our present intuitive understanding of the quark gluon structure of hadrons.

PDFs are defined as the forward matrix element of a light-like correlation func-

*Typeset names in $8 \mathrm{pt}$ roman, uppercase. Use the footnote to indicate the present or permanent address of the author.

†State completely without abbreviations, the affiliation and mailing address, including country. Typeset in 8 pt italic. 
tion, i.e.

$$
\begin{aligned}
q(x) & =\int \frac{d x^{-}}{4 \pi}\left\langle P, S\left|\bar{q}\left(-\frac{x^{-}}{2}, \mathbf{0}_{\perp}\right) \gamma^{+} q\left(\frac{x^{-}}{2}, \mathbf{0}_{\perp}\right)\right| P, S\right\rangle e^{i x p^{+} x^{-}} \\
\Delta q(x) S^{+} & =P^{+} \int \frac{d x^{-}}{4 \pi}\left\langle P, S\left|\bar{q}\left(-\frac{x^{-}}{2}, \mathbf{0}_{\perp}\right) \gamma^{+} \gamma_{5} q\left(\frac{x^{-}}{2}, \mathbf{0}_{\perp}\right)\right| P, S\right\rangle e^{i x p^{+} x^{-}} .
\end{aligned}
$$

Throughout this work, we will use light-cone gauge $A^{+}=0$. In all other gauges, a straight line gauge string connecting the quark field operators needs to be included in this definition (1). When sandwiched between states with the same light-cone momentum $p^{+}$, the operator

$$
\hat{O}_{q}\left(x, \mathbf{0}_{\perp}\right) \equiv \int \frac{d x^{-}}{4 \pi} \bar{q}\left(-\frac{x^{-}}{2}, \mathbf{0}_{\perp}\right) \gamma^{+} q\left(\frac{x^{-}}{2}, \mathbf{0}_{\perp}\right) e^{i x p^{+} x^{-}}
$$

in Eq. (11) has the effect of filtering out (the light-cone 'good' component of) quark fields with momentum fraction $x$, which is why PDFs 'measure' the light-cone momentum density of the quarks.

More recently, generalized parton distributions (GPDs) 1 have attrafted a considerable amount of interest (for a recent review, see for example Ref. 6 ), since it has been pointed out that on the one hand GPDs can be related to the total angular momentum carried by quarks in the nucleon and on the other hand they play an important role in deeply virtual Compton scattering experiments 3. GPDs are defined as matrix elements of the same operator that is used to define conventional PDFs, except that GPDs are defined as transition matrix elements between states with different momenta (and perhaps also different spins)

$$
\begin{aligned}
& \int \frac{d x^{-}}{4 \pi}\left\langle P^{\prime}, S^{\prime}\left|\bar{q}\left(-\frac{x^{-}}{2}, \mathbf{0}_{\perp}\right) \gamma^{+} q\left(\frac{x^{-}}{2}, \mathbf{0}_{\perp}\right)\right| P, S\right\rangle e^{i x \bar{p}^{+} x^{-}} \\
& \quad=\frac{1}{2 \bar{p}^{+}} \bar{u}\left(p^{\prime}, s^{\prime}\right)\left(\gamma^{+} H_{q}(x, \xi, t)+i \frac{\sigma^{+\nu} \Delta_{\nu}}{2 M} E_{q}(x, \xi, t)\right) u(p, s) \\
& \int \frac{d x^{-}}{4 \pi}\left\langle P^{\prime}, S^{\prime}\left|\bar{q}\left(-\frac{x^{-}}{2}, \mathbf{0}_{\perp}\right) \gamma^{+} \gamma_{5} q\left(\frac{x^{-}}{2}, \mathbf{0}_{\perp}\right)\right| P, S\right\rangle e^{i x \bar{p}^{+} x^{-}} \\
& \quad=\frac{1}{2 \bar{p}^{+}} \bar{u}\left(p^{\prime}, s^{\prime}\right)\left(\gamma^{+} \gamma_{5} \tilde{H}_{q}(x, \xi, t)+i \frac{\gamma_{5} \Delta^{+}}{2 M} \tilde{E}(x, \xi, t)\right) u(p, s)
\end{aligned}
$$

with $\bar{p}^{\mu}=\frac{1}{2}\left(p^{\mu}+p^{\prime \mu}\right)$ being the mean momentum of the target, $\Delta^{\mu}=p^{\prime \mu}-p^{\mu}$ the four momentum transfer, and $t=\Delta^{2}$ the invariant momentum transfer. The skewedness parameter $\xi=-\frac{\Delta^{+}}{2 \bar{p}^{+}}$quantifies the change in light-cone momentum.

GPDs allow for a unified description of a number of hadronic properties 3 ; for example:

1. In the forward limit they reduce to conventional PDFs

$$
\begin{aligned}
H_{q}(x, 0,0) & =q(x) \\
\tilde{H}_{q}(x, 0,0) & =\Delta q(x) .
\end{aligned}
$$


2. When one integrates GPDs over $x$ they reduce to the usual form factors, e.g. the Dirac form factors

$$
\begin{aligned}
& \sum_{q} e_{q} \int d x H_{q}(x, \xi, t)=F_{1}(t) \\
& \sum_{q} e_{q} \int d x E_{q}(x, \xi, t)=F_{2}(t),
\end{aligned}
$$

3. Similar relations exist for the so called 'generalized form factors'

$$
\begin{aligned}
& \sum_{q} e_{q}^{2} \int \frac{d x}{x} H_{q}(x, 0, t)=R_{V}(t) \\
& \sum_{q} e_{q}^{2} \int \frac{d x}{x} E_{q}(x, 0, t)=R_{V}(t) \\
& \sum_{q} e_{q}^{2} \int \frac{d x}{x} \tilde{H}_{q}(x, 0, t)=R_{A}(t) \\
& \sum_{q} e_{q}^{2} \int \frac{d x}{x} \tilde{E}_{q}(x, 0, t)=R_{P}(t),
\end{aligned}
$$

which play a role in (real) wide angle Compton scattering 4 .

4. And integrals like

$$
\mathcal{H}_{q}(\xi, t) \equiv \int_{-1}^{1} d x H_{q}(x, \xi, t)\left(\frac{1}{x+\xi-i \varepsilon}+\frac{1}{x-\xi+i \varepsilon}\right)
$$

describe the deeply virtual Compton scattering amplitude in the Bjorken scaling limit 3 .

Even though the abovementioned experiments provide only an indirect measurement of GPDs in the form of integrals (with the exception of the forward limit of GPDs, which is measured in DIS), it is clear that GPDs parameterize many hadronic property at the same time. The fact that they are connected to so many measurable quantities is not only the reason why they are of such central importance for hadron structure but at they same time provides a realistic hope that a combination of data from different classes of experiments together with enough theoretical constraints will eventually suffice to pin down these observables.

However, even though GPDs are such important observables, their physical interpretation is still rather obscure: since the initial and final states in Eqs. (3 (1) are not the same, GPDs in general do not have an immediate probabilistic interpretation as a density, which has made it very difficult to develop a simple physical interpretation for these observables.

${ }^{a}$ Note that the dependence on the longitudinal momentum transfer drops out due to Lorentz invariance in Eq. (6). 
Eq. (5) already points towards a very interesting connection between form factors and GPDs in the sense that GPDs provide a decomposition of the form factor with respect to the mean longitudinal momentum fraction $x=\left(k_{q}^{+}+k_{q}^{+^{\prime}}\right) / 2 \bar{P}^{+}$of the active quark, i.e. GPDs allow to determine how much quarks with a specific momentum fraction $x$ contribute to the form factor and therefore GPDs provide a (light-cone) momentum decomposition of the form factor. Therefore, it should be clear that knowledge of GPDs should be able to discriminate between different mechanisms for form factors at large momentum transfer.

However, there is an even more important analogy between form factors and GPDs: the form-factor, i.e. the non-forward matrix element of the current operator, describes how the charge (i.e. the forward matrix element of the same operator) is distributed in position space. 1 GPDs are the off-forward matrix elements of the operator $\hat{O}_{q}\left(x, \mathbf{0}_{\perp}\right)$ [Eq. (2)]. The forward matrix elements of $\hat{O}_{q}\left(x, \mathbf{0}_{\perp}\right)$ yield the usual PDFs. By analogy with form factors one would therefore expect that GPDs contain information about how the usual PDFs (the forward matrix elements) are distributed in position space 0 (Table 1). The mere fact that some kind of connec-

Table 1. Comparison between forward and off-forward matrix elements of the current operator as well as the operator that probes parton distribution functions. By analogy with form factors, one expects that GPDs contain information about how PDFs (the corresponding forward matrix elements) are distributed in position space.

\begin{tabular}{cccc}
\hline Operator & $\begin{array}{c}\text { forward matrix } \\
\text { element }\end{array}$ & $\begin{array}{c}\text { off-forward } \\
\text { matrix element }\end{array}$ & $\begin{array}{c}\text { position space } \\
\text { interpretation }\end{array}$ \\
\hline $\bar{q} \gamma^{+} q$ & $Q$ & $F(t)$ & $\rho(\vec{r})$ \\
$\int \frac{d x^{-} e^{i x p^{+} x^{-}}}{4 \pi} \bar{q}\left(\frac{-x^{-}}{2}\right) \gamma^{+} q\left(\frac{x^{-}}{2}\right)$ & $q(x)$ & $H_{q}(x, 0, t)$ & $q\left(x, \mathbf{b}_{\perp}\right)$ \\
\hline
\end{tabular}

tion between transverse positions of partons and the Fourier transform of GPDs with respect to the transverse momentum transfer might exist is evident. However, what is less obvious are important issues such as 'what exactly does one mean by the distribution of partons in the transverse plane', 'how is that transverse distribution related to GPDs', 'what are the limitations and corrections (e.g. relativistic corrections) to this position space interpretation', 'is there a strict probability interpretation', and 'what is the role of polarization'. The rest of the paper will be devoted to discussing this connection and and illustrating its consequences. Before doing so, it is worthwhile to discuss what kind of result one may anticipate. First of all, since a measurement of a parton distribution corresponds to a measurement of the momentum component of partons in the direction of the target's momentum. Because of the Heisenberg uncertainty principle, one should therefore not expect to

\footnotetext{
b Strictly speaking this is of course only true nonrelativistically as well as in specific frames (e.g. Breit frame), but this does not matter here since we use the connection $F(t) \longleftrightarrow \rho(\vec{r})$ here only as a motivation.
} 
be able to measure the 'longitudinal' position of partons. Measuring the transverse position simultaneously with the longitudinal momentum is not ruled out by the uncertainty principle, and therefore at the very best what one might expect to able to determine is the distribution of partons in the transverse plane. For this purpose, we will start out in the following by defining what we mean by impact parameter dependent parton distributions and then establish their connection to GPDs.

\section{Impact Parameter Dependent Parton Distribution}

In the case of nonrelativistic form factors, before one can introduce the notion of a charge distribution in position space, it is necessary to localize the target, e.g. by working in the center of mass frame. If one wants to talk about the distribution of partons in position space then

In order to be able to define impact parameter dependent PDFs one first needs to localize the nucleon in the transverse direction. For this purpose we introduce 9

$$
\left|p^{+}, \mathbf{R}_{\perp}=\mathbf{0}_{\perp}, \lambda\right\rangle \equiv \mathcal{N} \int \frac{d^{2} \mathbf{p}_{\perp}}{(2 \pi)^{2}}\left|p^{+}, \mathbf{p}_{\perp}, \lambda\right\rangle .
$$

where $\left|p^{+}, \mathbf{p}_{\perp}, \lambda\right\rangle$ are light-cone helicity eigenstates (see Appendix $A$ ) and $\mathcal{N}$ is a normalization factor satisfying $|\mathcal{N}|^{2} \int \frac{d^{2} \mathbf{p}_{\perp}}{(2 \pi)^{2}}=1$. This state is localized in the sense that its transverse center of momentum $\mathbf{R}_{\perp}$ is at the origin. For a state with total momentum $p^{+}$the transverse center of momentum is defined as

$$
\mathbf{R}_{\perp} \equiv \frac{1}{p^{+}} \int d x^{-} d^{2} \mathbf{x}_{\perp} \Theta^{++} \mathbf{x}_{\perp}
$$

where $\Theta^{\mu \nu}$ is the energy momentum tensor (Appendix A $)$. For practical purposes the intuitive parton representation for $\mathbf{R}_{\perp}$ is very useful

$$
\mathbf{R}_{\perp}=\sum_{i} x_{i} \mathbf{r}_{\perp, i} .
$$

The summation in Eq. (11) is over all partons in the hadron and $x_{i}$ is the momentum fraction carried by the $i^{\text {th }}$ partons in the infinite momentum frame. In summary, $\left|p^{+}, \mathbf{R}_{\perp}=\mathbf{0}_{\perp}, \lambda\right\rangle$ is a simultaneous eigenstate of $\hat{p}^{+}, \mathbf{R}_{\perp}$ and $J_{z}$, with eigenvalues $p^{+}, \mathbf{0}_{\perp}$ and $\lambda$ respectively.

Working with this localized state is very similar to working in the center of mass frame in nonrelativistic physics. The main difference being the weight factors, which are the mass fractions in the case of the nonrelativistic center of mass become momentum fractions in the transverse center of momentum. This is related to the properties of the Galilean subgroup of transverse boosts in the infinite momentum frame (Appendix A).

c Strictly speaking, we should work with wave packefs here in order to avoid states that are normalized to $\delta$ functions. This has been done in Ref. 0 and will not be repeated here because it makes the reasoning much more lengthy and less transparent. 
For such a transversely localized state, one can define an impact parameter dependent PDF, via ${ }^{\mathrm{d}}$

$$
q\left(x, \mathbf{b}_{\perp}\right) \equiv\left\langle p^{+}, \mathbf{R}_{\perp}=\mathbf{0}_{\perp}, \lambda\left|\hat{O}_{q}\left(x, \mathbf{b}_{\perp}\right)\right| p^{+}, \mathbf{R}_{\perp}=\mathbf{0}_{\perp}, \lambda\right\rangle,
$$

where $\hat{O}_{q}\left(x, \mathbf{b}_{\perp}\right)$ is obtained by a translation of Eq. (2) in the transverse plane, i.e.

$$
\hat{O}_{q}\left(x, \mathbf{b}_{\perp}\right)=\int \frac{d x^{-}}{4 \pi} \bar{q}\left(-\frac{x^{-}}{2}, \mathbf{b}_{\perp}\right) \gamma^{+} q\left(\frac{x^{-}}{2}, \mathbf{b}_{\perp}\right) e^{i x p^{+} x^{-}} .
$$

In gauges other than light cone gauge, a straight line gauge string connecting the points $\left(\frac{-x^{-}}{2}, \mathbf{b}_{\perp}\right)$ and $\left(\frac{x^{-}}{2}, \mathbf{b}_{\perp}\right)$ needs to be inserted in Eqs. (2) and (13).

In Section 2.2 we will show that these impact parameter dependent parton distributions satisfy positivity constraints which justifies a probabilistic interpretation. However, before that we will establish the connection between impact parameter dependent parton distributions and GPDs.

One may be tempted to expect a connection between impact parameter dependent PDFs, which are densities in transverse position space, and so called unintegrated parton densities $f\left(x, \mathbf{k}_{\perp}\right)$, which are densities in transverse momentum space. Even though there exist some inequalities, similar to the Heisenberg inequality, relating the width in transverse position space to the width in transverse momentum space, no direct connection exists between the two, unless one makes specific assumptions about the functional form of the hadron wave function. Strictly speaking, there just is no direct connection between momentum densities and position space densities in a many particle system and therefore there is also no direct connection between $f\left(x, \mathbf{k}_{\perp}\right)$ and $q\left(x, \mathbf{b}_{\perp}\right)$.

\subsection{Impact Parameter Dependent PDFs and GPDs}

In the following, we will focus on the case of purely transverse momentum transfer $\Delta^{+}=0$ i.e. $\xi=0$. Furthermore, we will start out by considering GPDs without helicity flip where only $H$ contributes in Eq. (3). Using $\bar{u}\left(p^{\prime}\right) \gamma^{+} u(p)=2 p^{+}$when $p^{+}=p^{+}$one thus finds

$$
\int \frac{d x^{-}}{4 \pi}\left\langle P^{\prime}, \lambda\left|\bar{q}\left(-\frac{x^{-}}{2}, \mathbf{0}_{\perp}\right) \gamma^{+} q\left(\frac{x^{-}}{2}, \mathbf{0}_{\perp}\right)\right| P, \lambda\right\rangle e^{i x p^{+} x^{-}}=H_{q}(x, t),
$$

where we introduced the shorthand notation $H_{q}(x, t) \equiv H_{q}(x, 0, t)$.

In order to illustrate the physics of $H_{q}(x, t)$, we take the definition of impact parameter dependent PDFs and expand the definition of the nucleon state in the center of momentum frame $\left|p^{+}, \mathbf{R}_{\perp}=\mathbf{0}_{\perp}, \lambda\right\rangle$ in a plane wave basis

$$
\begin{aligned}
q\left(x, \mathbf{b}_{\perp}\right) & \equiv\left\langle p^{+}, \mathbf{R}_{\perp}=\mathbf{0}_{\perp}, \lambda\left|\hat{O}_{q}\left(x, \mathbf{b}_{\perp}\right)\right| p^{+}, \mathbf{R}_{\perp}=\mathbf{0}_{\perp}, \lambda\right\rangle \\
& =|\mathcal{N}|^{2} \int \frac{d^{2} \mathbf{p}_{\perp}}{(2 \pi)^{2}} \int \frac{d^{2} \mathbf{p}_{\perp}^{\prime}}{(2 \pi)^{2}}\left\langle p^{+}, \mathbf{p}_{\perp}^{\prime}, \lambda\left|\hat{O}_{q}\left(x, \mathbf{b}_{\perp}\right)\right| p^{+}, \mathbf{p}_{\perp}, \lambda\right\rangle
\end{aligned}
$$

\footnotetext{
${ }^{\mathrm{d}}$ Note that for unpolarized parton distributions, it is irrelevant whether $\lambda=\uparrow$ or $\lambda=\downarrow$ in Eq. 12.
} 


$$
=|\mathcal{N}|^{2} \int \frac{d^{2} \mathbf{p}_{\perp}}{(2 \pi)^{2}} \int \frac{d^{2} \mathbf{p}_{\perp}^{\prime}}{(2 \pi)^{2}}\left\langle p^{+}, \mathbf{p}_{\perp}^{\prime}, \lambda\left|\hat{O}_{q}\left(x, \mathbf{0}_{\perp}\right)\right| p^{+}, \mathbf{p}_{\perp} \lambda\right\rangle e^{i \mathbf{b}_{\perp} \cdot\left(\mathbf{p}_{\perp}-\mathbf{p}_{\perp}^{\prime}\right)} e^{i x p^{+} x^{-}},
$$

where the phase factor in the last step is due to the transverse translation, i.e. $\left\langle p^{+}, \mathbf{p}_{\perp}^{\prime}\left|\hat{O}_{q}\left(x, \mathbf{b}_{\perp}\right)\right| p^{+}, \mathbf{p}_{\perp}\right\rangle=\left\langle p^{+}, \mathbf{p}_{\perp}^{\prime}\left|\hat{O}_{q}\left(x, \mathbf{0}_{\perp}\right)\right| p^{+}, \mathbf{p}_{\perp}\right\rangle e^{i \mathbf{b}_{\perp} \cdot\left(\mathbf{p}_{\perp}-\mathbf{p}_{\perp}^{\prime}\right)}$. The kind of matrix elements that appear in Eq. (15) are identical to matrix elements that appear in the definition of $H_{q}(x, 0, t)$. Combining Eqs. (15) and (14) one thus finds

$$
\begin{aligned}
q\left(x, \mathbf{b}_{\perp}\right) & =|\mathcal{N}|^{2} \int \frac{d^{2} \mathbf{p}_{\perp}}{(2 \pi)^{2}} \int \frac{d^{2} \mathbf{p}_{\perp}^{\prime}}{(2 \pi)^{2}} H_{q}\left(x,-\left(\mathbf{p}_{\perp}-\mathbf{p}_{\perp}^{\prime}\right)^{2}\right) e^{i \mathbf{b}_{\perp} \cdot\left(\mathbf{p}_{\perp}-\mathbf{p}_{\perp}^{\prime}\right)} \\
& =\int \frac{d^{2} \boldsymbol{\Delta}_{\perp}}{(2 \pi)^{2}} H_{q}\left(x,-\boldsymbol{\Delta}_{\perp}^{2}\right) e^{-i \mathbf{b}_{\perp} \cdot \boldsymbol{\Delta}_{\perp}}
\end{aligned}
$$

where we switched in the last step to total and relative transverse momentum, i.e. $\int d^{2} \mathbf{p}_{\perp} \int d^{2} \mathbf{p}_{\perp}^{\prime}=\int d^{2} \boldsymbol{\Delta}_{\perp} \int d^{2} \overline{\mathbf{p}}_{\perp}$, with $\overline{\mathbf{p}}_{\perp}=\frac{1}{2}\left(\mathbf{p}_{\perp}+\mathbf{p}_{\perp}^{\prime}\right)$ and $\boldsymbol{\Delta}_{\perp}=\mathbf{p}_{\perp}^{\prime}-\mathbf{p}_{\perp}$, and used the fact that $H_{q}$ did not depend on $\overline{\mathbf{p}}_{\perp}$. . $^{-}$

This proves that the impact parameter dependent PDF defined in Eq. (12) is the Fourier transform of $H_{q}\left(x,-\boldsymbol{\Delta}_{\perp}^{2}\right)$, i.e. if one knows $H_{q}\left(x,-\boldsymbol{\Delta}_{\perp}^{2}\right)$ one can determine the distribution of partons simultaneously as a function of the light-cone momentum fraction $x$ and the distance $\mathbf{b}_{\perp}$ from the transverse center of momentum. This very important result was already obtained in Ref. 6 , where it was shown that working with wave packets in $\perp$ momentum space, i.e. replacing $\int d^{2} \mathbf{p}_{\perp}$ by $\int d^{2} \mathbf{p}_{\perp} \psi\left(\mathbf{p}_{\perp}\right)$, where $\psi\left(\mathbf{p}_{\perp}\right)$ is a slowly varying function of $\mathbf{p}_{\perp}$ that is taken to be a constant towards the end of the calculation, does not change the final result. F It only leads to much more complicated intermediate expressions. Here we followed a much more simplified derivation 10 using non-normalizable states in order to better illustrate the physics of the result.

As a side remark, it should be emphasized that the interpretation of the Fourier transform of form factors as charge densities is, in the rest frame, spoiled by relativistic normalization factors as well as the effect of Lorentz contraction. It is also well known that these relativistic corrections are not present in special frames, such as for example the Breit frame. Our derivation above shows that such corrections not present in the infinite momentum frame either, i.e. the identification of the Fourier transform of $H_{q}\left(x, 0,-\boldsymbol{\Delta}^{2}\right)$ w.r.t. $\boldsymbol{\Delta}$ as a distribution of partons in transverse position space is not limited by relativistic effects. As a corollary, since $\sum_{q} e_{q} \int d x H_{q}\left(x, 0,-\boldsymbol{\Delta}^{2}\right)=F_{1}\left(-\boldsymbol{\Delta}^{2}\right)$, this means that the Fourier transform of $F_{1}\left(-\Delta^{2}\right)$ can be interpreted as the charge distribution in the infinite momentum

e Note that the last property, i.e. the fact that $H_{q}\left(x, 0,-\Delta_{\perp}^{2}\right.$ ) does not depend on $\overline{\mathbf{p}}_{\perp}$ (which reflects the Galilei invariance under $\perp$ boosts in the IMF), is one of the crucial ingredients of our derivation!

${ }^{\mathrm{f}}$ If one includes a factor $(1-x)$ in the exponent of the Fourier transform in Eq. (16), one obtains the distribution not as a function of the distance of the active quark from the $\mathrm{CM}$ of the whole hadron but as a function of the distancy to the CM of the spectators.

${ }^{\mathrm{g}}$ This result was also confirmed in Ref. $\mathrm{l}$, where Gaussian wave packets were used. 
frame as a function of the transverse distance from the (transverse) center of momentum. This interpretation, together with the observation that this interpretation is not spoiled by relativistic corrections, are both surprisingly little known results.

\subsection{Density Interpretation for Impact Parameter Dependent Parton Distributions}

In this section, we will show that $q\left(x, \mathbf{b}_{\perp}\right)$ satisfies positivity constraints, which allows one to associate a probabilistic interpretation. For this purpose we note that only the projection on the 'good' quark field component $\psi_{(+)} \equiv \frac{1}{2} \gamma^{-} \gamma^{+} \psi$ contributes in twist-2 matrix elements, i.e. $\bar{\psi}^{\prime} \gamma^{+} \psi=\bar{\psi}_{(+)}^{\prime} \gamma^{+} \psi_{(+)}$.

When one quantizes fermions on the light-cone (or in the infinite momentum frame) only this 'good' component is dynamical and an expansion in terms of canonical raising and lowering operators reads 11

$$
\begin{array}{r}
\psi_{(+)}\left(x^{-}, \mathbf{x}_{\perp}\right)=\int_{0}^{\infty} \frac{d k^{+}}{\sqrt{4 \pi k^{+}}} \int \frac{d^{2} \mathbf{k}_{\perp}}{2 \pi} \sum_{s}\left[u_{(+)}(k, s) b_{s}\left(k^{+}, \mathbf{k}_{\perp}\right) e^{-i k x}\right. \\
\left.+v_{(+)}(k, s) d_{s}^{\dagger}\left(k^{+}, \mathbf{k}_{\perp}\right) e^{i k x}\right],
\end{array}
$$

where $b$ and $d$ satisfy the usual (canonical) equal light-cone time $x^{+}$anti-commutation relations, e.g.

$$
\left\{b_{r}\left(k^{+}, \mathbf{k}_{\perp}\right), b_{s}^{\dagger}\left(q^{+}, \mathbf{q}_{\perp}\right)\right\}=\delta\left(k^{+}-q^{+}\right) \delta\left(\mathbf{k}_{\perp}-\mathbf{q}_{\perp}\right) \delta_{r s}
$$

and the normalization of the spinors is such that

$$
\bar{u}_{(+)}(p, r) \gamma^{+} u_{(+)}(p, s)=2 p^{+} \delta_{r s} .
$$

An explicit representation for these spinors can be found in Ref. $\mathrm{G}$. We now insert these expansions into our definition of $q\left(x, \mathbf{b}_{\perp}\right)$. Using $\bar{u}_{(+)}\left(p^{\prime}, r\right) \gamma^{+} u_{(+)}(p, s)=$ $2 p^{+} \delta_{r s}$, when $p^{+}={p^{+}}^{\prime}$, one finds for $x>0$

$$
\begin{aligned}
q\left(x, \mathbf{b}_{\perp}\right)= & \mathcal{N}^{\prime} \sum_{s} \int \frac{d^{2} \mathbf{k}_{\perp}}{2 \pi} \int \frac{d^{2} \mathbf{k}_{\perp}^{\prime}}{2 \pi} e^{-i \mathbf{b}_{\perp} \cdot\left(\mathbf{k}_{\perp}^{\prime}-\mathbf{k}_{\perp}\right)} \\
& \left\langle p^{+}, \mathbf{R}_{\perp}=\mathbf{0}_{\perp}, \lambda\left|b_{s}^{\dagger}\left(x p^{+}, \mathbf{k}_{\perp}^{\prime}\right) b_{s}\left(x p^{+}, \mathbf{k}_{\perp}\right)\right| p^{+}, \mathbf{R}_{\perp}=\mathbf{0}_{\perp}, \lambda\right\rangle .
\end{aligned}
$$

Note that we made use of the fact that the states that appear in the 'initial' and 'final' states of Eq. (20) have the same $p^{+}$and therefore both the $k^{+}$of the lowering and the raising operator must be the same (i.e. both are equal to $x p^{+}$), which allows one to replace in the matrix element

$$
\int \frac{d x^{-}}{4 \pi} \bar{q}\left(0^{-}, \mathbf{b}_{\perp}\right) \gamma^{+} q\left(x^{-}, \mathbf{b}_{\perp}\right) e^{i x^{-} p^{+} x} \quad \longrightarrow \quad \mathcal{N}^{\prime} \tilde{b}^{\dagger}\left(x p^{+}, \mathbf{b}_{\perp}\right) \tilde{b}\left(x p^{+}, \mathbf{b}_{\perp}\right),
$$

where $\mathcal{N}^{\prime}$ is a constant, which is, in the infinite volume proportional to $\delta(0)$. For $x<0$ one finds a similar expression with $b_{s}^{\dagger} b_{s}$ replaced by $-d_{s} d_{s}^{\dagger}$.

$\overline{\mathrm{h} \text { The 'bad' component } \psi_{(-)} \equiv \frac{1}{2} \gamma^{+} \gamma^{-}} \psi$ satisfies a constraint equation. 
In order to simplify these expressions further, we introduce a 'hybrid' representation, with field operators that are labeled by $\perp$ position space and longitudinal momentum space variables, i.e. we introduce

$$
\tilde{b}\left(k^{+}, \mathbf{x}_{\perp}\right) \equiv \int \frac{d^{2} \mathbf{k}_{\perp}}{2 \pi} b\left(k^{+}, \mathbf{k}_{\perp}\right) e^{i \mathbf{k}_{\perp} \cdot \mathbf{x}_{\perp}}
$$

and likewise for $\tilde{d}^{\dagger}$. Inserting $\tilde{b}$ into Eq. (20) one easily finds

$$
\begin{aligned}
q\left(x, \mathbf{b}_{\perp}\right) & \sim \sum_{s}\left\langle p^{+}, \mathbf{R}_{\perp}=\mathbf{0}_{\perp}, \lambda\left|\tilde{b}_{s}^{\dagger}\left(x p^{+}, \mathbf{b}_{\perp}\right) \tilde{b}_{s}\left(x p^{+}, \mathbf{b}_{\perp}\right)\right| p^{+}, \mathbf{R}_{\perp}=\mathbf{0}_{\perp}, \lambda\right\rangle \\
& \left.=\sum_{s}\left|\tilde{b}_{s}\left(x p^{+}, \mathbf{b}_{\perp}\right)\right| p^{+}, \mathbf{R}_{\perp}=\mathbf{0}_{\perp}, \lambda\right\rangle\left.\right|^{2} \geq 0,
\end{aligned}
$$

for $x<0$. Similarly, one finds for $x<0$

$$
\left.q\left(x, \mathbf{b}_{\perp}\right) \sim-\sum_{s}\left|\tilde{d}_{s}^{\dagger}\left(x p^{+}, \mathbf{b}_{\perp}\right)\right| p^{+}, \mathbf{R}_{\perp}=\mathbf{0}_{\perp}, \lambda\right\rangle\left.\right|^{2} \leq 0 .
$$

These results were first shown in Ref. 10 and confirmed in Ref. 9 .

Because of these positivity (negativity) constraints, it is legitimate to associate the physical interpretation of a probability density distribution with the impact parameter dependent parton distribution $q\left(x, \mathbf{b}_{\perp}\right)$. The fact that the impact parameter dependent parton distributions have a density interpretation is very important because it shows that they have a physical significance beyond being the Fourier transforms of GPDs!

\subsection{Modeling Impact Parameter Dependent Parton Distributions}

GPDs cannot be uniquely determined from Compton scattering experiments alone, where they appear in the integrand of convolution integrals. Until sufficient constraints from theory and/or other experiments exist, that allow one to pin down GPDs uniquely, one may still attempt to extract GPDs from (virtual) Compton scattering data by making a physically motivated ansatz for the GPDs, where the free parameters in the ansatz are determined by fitting to the data. Since such a procedure is clearly model dependent, it is desirable to use as many general theoretical constraints as possible in order to minimize the dependence on specific model features. The results from this paper can be used to provide some general theoretical constraints as we will discuss in the following.

First of all, in order to be consistent with the above positivity constraints [(23) and (24)], all realistic Ansätze for $H_{q}(x, 0, t)$ should satisfy

$$
\begin{array}{ll}
\int d^{2} \boldsymbol{\Delta}_{\perp} e^{-i \mathbf{b}_{\perp} \cdot \boldsymbol{\Delta}_{\perp}} H_{q}\left(x, 0,-\boldsymbol{\Delta}_{\perp}^{2}\right) \geq 0 & \text { for } \quad x>0 \\
\int d^{2} \boldsymbol{\Delta}_{\perp} e^{-i \mathbf{b}_{\perp} \cdot \boldsymbol{\Delta}_{\perp}} H_{q}\left(x, 0,-\boldsymbol{\Delta}_{\perp}^{2}\right) \leq 0 & \text { for } \quad x<0
\end{array}
$$

for any value of $\mathbf{b}_{\perp}$ and any value of $x$. Of course, many Ansätze, especially those with a Gaussian $t$ dependence, are already consistent with these new constraints. 
However, additional constraints arise due to the fact that GPDs can be linked to impact parameter dependent PDFs.

First of all, the mere fact that the impact parameter dependent PDFs are measured with respect to the transverse center of momentum $\mathbf{R}_{\perp}=\sum_{i} x_{i} \mathbf{r}_{\perp, i}$ implies that $H_{q}(x, 0, t)$ should become $t$-independent as $x \rightarrow 1$. The reason for this behavior is very simple: the transverse center of momentum of a hadron is defined as the weighted average of transverse positions of all partons in the hadron, where the weight factors are the momentum fractions carried by each parton. Since $x$ in $H_{q}(x, 0, t)$ is the momentum fraction carried by the active quark, this active quark carries all the momentum of the hadron when one takes $x \rightarrow 1$ and therefore the contribution from all partons other than the active quark in Eq. (11) becomes negligible. Hence the active quark is always very close to the transverse center of momentum when $x \rightarrow 1$ and the impact parameter dependent $\operatorname{PDF} q\left(x, \mathbf{b}_{\perp}\right)$ has a very peaked 'transverse profile' in this limit. More precisely, the transverse width of the impact parameter dependent PDF

$$
\left\langle R_{\perp}^{2}(x)\right\rangle \equiv \frac{\int d^{2} \mathbf{b}_{\perp} \mathbf{b}_{\perp}^{2} q\left(x, \mathbf{b}_{\perp}\right)}{\int d^{2} \mathbf{b}_{\perp} q\left(x, \mathbf{b}_{\perp}\right)}
$$

should vanish as $x \rightarrow 1$. Thus $H_{q}\left(x, 0,-\boldsymbol{\Delta}_{\perp}^{2}\right)$ (being the Fourier transform of $\left.q\left(x, \mathbf{b}_{\perp}\right)\right)$ should become independent of $\boldsymbol{\Delta}_{\perp}^{2}$ as $x \rightarrow 1$. We should emphasize that this behavior is consistent with the 'Feynman picture' for hadron form factors near $x=1$ 12. In this picture, configurations where one parton carries $x \approx 1$ and all others are 'wee' can be easily scattered elastically (by striking the parton with $x \approx 1$ ) since the wee partons do not have a direction and thus can be easily 'turned around'. Therefore, in the Feynman picture, contributions to the form factor where the active quark carries a larger fraction of the hadron's total momentum tend to have a weaker $t$ dependence.

The constraint that $H_{q}(x, 0, t)$ should become $t$ independent as $x \rightarrow 1$ rules out factorized Ansätze of the form

$$
H_{q}(x, 0, t)=q(x) \cdot F(t),
$$

since they do not become $t$ independent near $x=1$.

The position space interpretation of GPDs also allows one to put constraints on the behavior near $x=0$. Space time descriptions of hadron structure ? suggest that the transverse size of hadrons should grow like $\alpha \ln \frac{1}{x}$ near $x=0$, where $\alpha$ is some constant. In fact, a more rapid growth (e.g. power law) of the transverse size for $x \rightarrow 0$ would also lead to power law growth of total cross sections and would therefore lead to violations of unitarity bounds.

Because of the connection between the transverse size and the slope of $H_{q}(x, 0, t)$,

${ }^{\mathrm{i}}$ This is very similar to $B$-mesons, where the $b$ quark does not recoil very much and therefore has a very localized distribution in position space. 
this result implies for example that an ansatz like

$$
H_{q}\left(x, 0,-\boldsymbol{\Delta}_{\perp}^{2}\right)=q(x) e^{-a \Delta_{\perp}^{2} \frac{1-x}{x}},
$$

is inconsistent with abovementioned constraints about the transverse size of hadrons near $x=0$. [

An improved Gaussian ansatz for $H_{q}\left(x, 0,-\Delta_{\perp}^{2}\right)$, which is consistent with both the $x \rightarrow 1$ and $x \rightarrow 0$ constraints, is given by

$$
H_{q}\left(x, 0,-\Delta_{\perp}^{2}\right)=q(x) e^{-a \Delta_{\perp}^{2} \ln \frac{1}{x}}
$$

or

$$
H_{q}\left(x, 0,-\boldsymbol{\Delta}_{\perp}^{2}\right)=q(x) e^{-a \Delta_{\perp}^{2}(1-x) \ln \frac{1}{x}} .
$$

The latter ansatz yields at the same time an asymptotic form factor that is consistent with quark counting: if the end-point behavior of the PDF is taken such that $q(x) \sim(1-x)^{2 n_{s}-1}$ near $x=1$ then the large $Q^{2}$ power law behavior of the form factor is $F\left(Q^{2}\right) \sim\left(\frac{1}{Q^{2}}\right)^{n_{s}}$.

Although the above discussion may appear purely academic at the present stage, where very little information on GPDs exists, it may still have some phenomenological significance because in Compton scattering the contributions from the small $x$ region are enhanced due to the factor $1 / x$ (7). The ansatz in Eq. (28) leads to a much more rapid suppression of contributions from the small $x$ region at larger values of $t$ than a logarithmic dependence on $x$ in the exponent [Eqs. (29) and (30)].

In order to gain some intuitive understanding about the behavior of impact parameter dependent parton distributions, we transform Eq. (30) to impact parameter space, where we find

$$
q\left(x, \mathbf{b}_{\perp}\right)=q(x) \frac{1}{4 \pi a(1-x) \ln \frac{1}{x}} e^{-\frac{\mathbf{b}_{\perp}^{2}}{4 a(1-x) \ln \frac{1}{x}}} .
$$

The result, which is depicted in Fig. 1, exhibits the general features that we described above. First, there is a general decrease in magnitude with increasing $x$, which arises mostly due to the decrease of $q(x)$. Furthermore, one can also a notice a decreasing transverse width of the distribution $q\left(x, \mathbf{b}_{\perp}\right)$ as $x$ increases, which is in accord with our general prediction. For $x \rightarrow 1$ the transverse width goes to zero.

\section{Nucleon Helicity Flip Distributions}

So far we have considered only two out of eight GPDs 3 . In order to investigate the interpretation of the other six GPDs, it is necessary to consider amplitudes

${ }^{\mathrm{j}}$ Of course, for intermediate and large values of $x$, this ansatz may nevertheless be a useful approximation to the actual behavior and this ansatz also satisfies our above constraint that $H_{q}(x, 0, t)$ should become $t$ independent near $x=1$. 


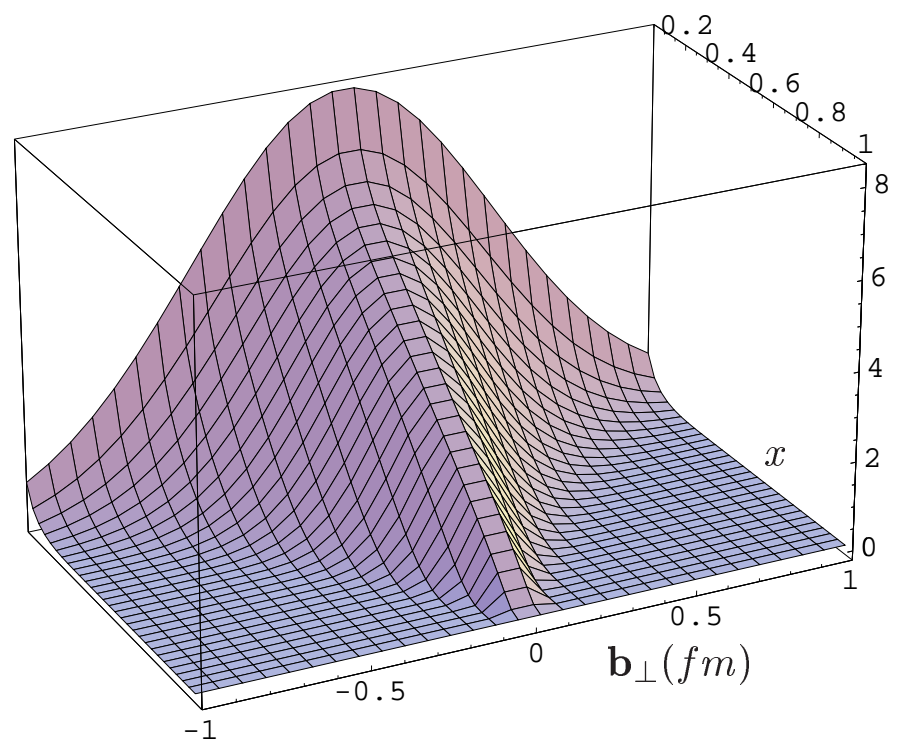

Fig. 1. Impact parameter dependent parton distribution $u\left(x, \mathbf{b}_{\perp}\right)$ for the simple model (31).

where either the nucleon or quark helicity (or both) flip, which makes it much more difficult to develop a probabilistic interpretation. Therefore, we focus here on $E(x, 0, t)$, which appears in amplitudes where the nucleon helicity flips and the quark helicity does not flip

$$
\int \frac{d x^{-}}{4 \pi} e^{i p^{+} x^{-} x}\left\langle P+\Delta, \uparrow\left|\bar{q}(0) \gamma^{+} q\left(x^{-}\right)\right| P, \downarrow\right\rangle=-\frac{\Delta_{x}-i \Delta_{y}}{2 M} E\left(x, 0,-\Delta_{\perp}^{2}\right)
$$

For a probabilistic interpretation, it is necessary to consider amplitudes where the initial and final states are the same. Since $E(x, 0, t)$ does not contribute when the initial and final state have the same helicity, we will now consider a state that is a superposition of (transversely localized) nucleon states with opposite helicities. For brevity, we will denote this state as $|X\rangle$, i.e.

$$
|X\rangle \equiv \frac{1}{\sqrt{2}}\left(\left|p^{+}, \mathbf{R}_{\perp}=\mathbf{0}_{\perp}, \uparrow\right\rangle+\left|p^{+}, \mathbf{R}_{\perp}=\mathbf{0}_{\perp}, \downarrow\right\rangle\right) .
$$

It is tempting to interpret this state as a state that is polarized in the $x$ direction and if we were dealing with a nonrelativistic system this would certainly be the case. However, for relativistic systems, spins are not invariant under boosts and one has to be careful here with such an interpretation. Nevertheless, let us proceed here and study the (unpolarized) impact parameter dependent PDF in this state

$$
\begin{aligned}
q_{X}\left(x, \mathbf{b}_{\perp}\right) & \equiv\left\langle X\left|\hat{O}_{q}\left(x, \mathbf{b}_{\perp}\right)\right| X\right\rangle \\
& =\int \frac{d^{2} \boldsymbol{\Delta}_{\perp}}{(2 \pi)^{2}} e^{-i \boldsymbol{\Delta}_{\perp} \cdot \mathbf{b}_{\perp}}\left[H_{q}\left(x, 0,-\boldsymbol{\Delta}_{\perp}^{2}\right)+i \frac{\Delta_{y}}{2 M} E_{q}\left(x, 0,-\boldsymbol{\Delta}_{\perp}^{2}\right)\right]
\end{aligned}
$$




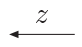

a)

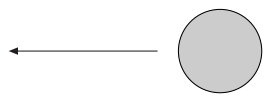

b)

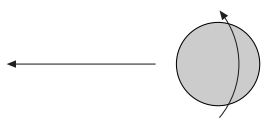

c)

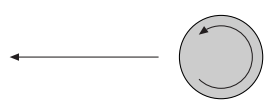

Fig. 2. Comparison of a non-rotating sphere that moves in the $z$ direction with a sphere that spins at the same time around the $z$ axis and a sphere that spins around the $x$ axis When the sphere spins around the $x$ axis, the rotation changes the distribution of momenta in the $z$ direction (adds/subtracts to velocity for $y>0$ and $y<0$ respectively). For the nucleon the resulting modification of the (unpolarized) momentum distribution is described by $E(x, 0, t)$.

$$
=q\left(x, \mathbf{b}_{\perp}\right)-\frac{1}{2 M} \frac{\partial}{\partial b_{y}} \mathcal{E}_{q}\left(x, \mathbf{b}_{\perp}\right),
$$

where we denoted the Fourier transform of $E\left(x, 0,-\boldsymbol{\Delta}_{\perp}^{2}\right)$ by $\mathcal{E}\left(x, \mathbf{b}_{\perp}\right)$, i.e.

$$
\mathcal{E}_{q}\left(x, \mathbf{b}_{\perp}\right) \equiv \int \frac{d^{2} \boldsymbol{\Delta}_{\perp}}{(2 \pi)^{2}} e^{-i \boldsymbol{\Delta}_{\perp} \cdot \mathbf{b}_{\perp}} E_{q}\left(x, 0,-\boldsymbol{\Delta}_{\perp}^{2}\right) .
$$

The physical significance of $\mathcal{E}\left(x, \mathbf{b}_{\perp}\right)$ is as follows. First, integration over both $x$ and $\mathbf{b}_{\perp}$ yields the contribution from quark flavor $q$ to the anomalous magnetic moment

$$
\int_{-1}^{1} d x \int d^{2} \mathbf{b}_{\perp} \mathcal{E}_{q}\left(x, \mathbf{b}_{\perp}\right)=F_{2, q}(0)=\kappa_{q}
$$

Secondly, Eq. (34) describes the transverse distortion of the unpolarized impact parameter dependent PDF if the state is not polarized in the $\pm z$ direction but rather in a transverse direction. The physics of this result can be illustrated by some classical example (Fig. 2) Consider a sphere that moves in the $z$ direction. The analog of PDFs in this example are the distributions of momenta in the $z$ direction from particles on the sphere as seen by an observer at rest. The distribution of these momenta in the transverse plane is the same regardless whether the sphere is nonrotating or spinning around the $z$ axis (in either direction) because spinning 


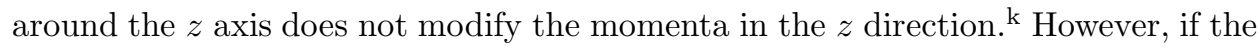
sphere spins around the $x$-axis while still moving along the $z$-axis, the longitudinal momentum distribution of particles on the sphere as seen by an observer at rest changes dependent on the transverse position of the observer since on one side of the $z$ axis the rotational motion adds to the translatory motion and on the other side it subtracts.

Of course, the nucleon is not just a rotating sphere but this simple example illustrate the physics of why the parton distribution in the transverse plane gets distorted and is no longer invariant under rotations around the $z$-axis when the nucleon is not longitudinally polarized. And in the nucleon it is the Fourier transform of $E\left(x, 0,-\Delta_{\perp}^{2}\right)$, which describes this distortion.

The second moment of $q_{X}\left(x, \mathbf{b}_{\perp}\right)$, i.e. $\int_{-1}^{1} d x x q_{X}\left(x, \mathbf{b}_{\perp}\right)$, is of particular interest since it describes how the momentum is distributed in the transverse plane

$$
\int_{-1}^{1} d x x q_{X}\left(x, \mathbf{b}_{\perp}\right)=\int_{-1}^{1} d x x q\left(x, \mathbf{b}_{\perp}\right)-\frac{\partial}{\partial b_{x}} \int_{-1}^{1} d x x \mathcal{E}_{q}\left(x, \mathbf{b}_{\perp}\right),
$$

i.e. the second moment of $\mathcal{E}_{q}$ describes how the distribution of momentum carried by quarks of flavor $q$ in the transverse plane changes when the nucleon is not polarized in the $z$ direction. In particular, the contribution from flavor $q$ to the transverse center of momentum in the "transversely polarized" state $|X\rangle$ is shifted away from the origin by

$$
\begin{aligned}
\int_{-1}^{1} d x \int d^{2} \mathbf{b}_{\perp} b_{y} x q_{X}\left(x, \mathbf{b}_{\perp}\right) & =-\int_{-1}^{1} d x \int d^{2} \mathbf{b}_{\perp} b_{y} x \frac{1}{2 M} \frac{\partial}{\partial b_{y}} \mathcal{E}_{q}\left(x, \mathbf{b}_{\perp}\right) \\
& =\int_{-1}^{1} d x \int d^{2} \mathbf{b}_{\perp} x \frac{1}{2 M} \mathcal{E}_{q}\left(x, \mathbf{b}_{\perp}\right) \\
& =\frac{1}{2 M} \int_{-1}^{1} d x x E_{q}(x, 0,0) .
\end{aligned}
$$

Therefore, the second moment of $E_{q}(x, 0,0)$ describes, in units of $\frac{1}{2 M}$, by how much the transverse center of momentum of quarks of flavor $q$ is shifted away from the origin in the 'transversely polarized' state $|X\rangle$. Of course, when summed over all partons (all quarks and gluons) the transverse center of momentum of the state $|X\rangle$ is still at the origin since it is a superposition of eigenstates of $\mathbf{R}_{\perp}$ with eigenvalue $\mathbf{0}_{\perp}$, i.e.

$$
\sum_{i \in q, g} \int_{-1}^{1} d x x E_{i}(x, 0,0)=0,
$$

which is nothing but the statement that the total (i.e. all flavors plus glue) anomalous gravito-magnetic moment has to vanish 14 .

\footnotetext{
kOne may also take this example as a simple illustration as to why the (unpolarized) impact
} parameter dependent PDFs are the same for nucleons with $\lambda=\uparrow$ and $\lambda=\downarrow$. 
If the center of momentum for quarks of flavor $q$ is shifted away from the origin in the $y$ direction then those quarks carry orbital angular momentum in the $x$ direction. Starting from this observation, one can obtain a more physical understanding of why the $2^{\text {nd }}$ moment of $E$ appears in Ji's angular momentum sum rule 20 .

Another important consequence can be derived from the fact that $q_{X}\left(x, \mathbf{b}_{\perp}\right)$, i.e. the unpolarized impact parameter dependent quark distribution in the state $|X\rangle$ has a probabilistic interpretation as well

$$
\begin{array}{ll}
q_{X}\left(x, \mathbf{b}_{\perp}\right) \geq 0 & \text { for } \quad x>0 \\
q_{X}\left(x, \mathbf{b}_{\perp}\right) \leq 0 & \text { for } \quad x<0 .
\end{array}
$$

This implies the inequality

$$
q\left(x, \mathbf{b}_{\perp}\right) \geq \frac{1}{2 M}\left|\frac{\partial}{\partial b_{i}} \mathcal{E}_{q}\left(x, \mathbf{b}_{\perp}\right)\right| .
$$

The physics of this result is that although the probability distribution of partons in the transverse plane gets distorted when the nucleon is polarized in a transverse direction, the resulting probability density needs to remain positive. Since the distortion is described by the gradient of $\mathcal{E}\left(x, \mathbf{b}_{\perp}\right)$, this sets some upper bound on the magnitude of $\nabla_{\perp} \mathcal{E}$. By considering more general helicity combinations one can derive inequalities connecting chirally even and odd parton distributions in impact parameter representation 15 , but we will not consider those here since chirally odd GPDs are even harder to measure than chirally even ones.

In order to illustrate the magnitude of the transverse distortion effect, we consider a simple model

$$
\begin{aligned}
& E_{u}(x, 0, t)=\frac{1}{2} \kappa_{u} H_{u}(x, 0, t) \\
& E_{d}(x, 0, t)=\kappa_{d} H_{d}(x, 0, t),
\end{aligned}
$$

where $H_{u}(x, 0, t)=2 H_{d}(x, 0, t)$ is taken from Eq. (30) and $\kappa_{u}$ and $\kappa_{d}$ are determined by making the approximation that only $u$ and $d$ quarks contribute to the nucleon's magnetic moment, i.e.

$$
\begin{aligned}
\kappa_{p} & =\frac{2}{3} \kappa_{u}-\frac{1}{3} \kappa_{n}=1.793 \\
\kappa_{n} & =\frac{2}{3} \kappa_{d}-\frac{1}{3} \kappa_{u}=-1.913 \\
\kappa_{u} & =2 \kappa_{p}+\kappa_{n}=1.673 \\
\kappa_{d} & =2 \kappa_{n}+\kappa_{p}=-2.033 .
\end{aligned}
$$

With $\int_{-1}^{1} d x H_{d}(x, 0,0)=1$ and $\int_{-1}^{1} d x H_{u}(x, 0,0)=2$ this ansatz satisfies $\int_{-1}^{1} d x E_{u}(x, 0,0)=\kappa_{u}$ and $\int_{-1}^{1} d x E_{d}(x, 0,0)=\kappa_{d}$. The resulting distributions in impact parameter space for both unpolarized and 'transversely polarized' protons are depicted in Figs. 3 and 0 for $u$ and $d$ quarks respectively. 
The above Ansatz for $E_{q}(x, 0, t)$ (43), together with our model for the impact parameter distribution of quarks in a longitudinally polarized nucleon (31), therefore yields for the distributions $q_{X}\left(x, \mathbf{b}_{\perp}\right)$ in a 'transversely polarized' nucleon

$$
\begin{aligned}
& u_{X}\left(x, \mathbf{b}_{\perp}\right)=\left[1+\frac{\kappa_{u} S_{x} b_{y}}{8 M a(1-x) \ln \frac{1}{x}}\right] u(x) \frac{1}{4 \pi a(1-x) \ln \frac{1}{x}} e^{-\frac{\mathbf{b}_{\perp}^{2}}{4 a(1-x) \ln \frac{1}{x}}} \\
& d_{X}\left(x, \mathbf{b}_{\perp}\right)=\left[1+\frac{\kappa_{d} S_{x} b_{y}}{4 M a(1-x) \ln \frac{1}{x}}\right] d(x) \frac{1}{4 \pi a(1-x) \ln \frac{1}{x}} e^{-\frac{\mathbf{b}_{\perp}^{2}}{4 a(1-x) \ln \frac{1}{x}}}
\end{aligned}
$$

where $S_{x}= \pm 1$ for $|X\rangle=\frac{1}{\sqrt{2}}(|\uparrow\rangle \pm|\downarrow\rangle)$ [Figs. 3 and 4 ]. One can make several observations from Figs. 3 and 1 . First of all, the distortion for $u$ and $d$ quarks is in opposite directions. In our model this results from the simple fact that $\kappa_{u}$ and $\kappa_{d}$ have opposite signs. However, since $\int_{-1}^{1} d x \int d^{2} \mathbf{b}_{\perp} \mathcal{E}_{q}\left(x, \mathbf{b}_{\perp}\right)=\kappa_{q}$ should always hold, one expects the fact that $u$ and $d$ quarks get distorted in opposite directions for a 'transversely polarized' state to be a more general phenomenon. Physically, one can understand the difference in signs by replacing the rigid rotation of the sphere in Fig. 2 by the intrinsic orbital angular momentum of the quarks, which may be either parallel or anti-parallel to the nucleon spin.

Secondly, one observes that the sideways distortion for $d$ quarks is about twice as strong as for $u$ quarks, even though $\kappa_{u}$ and $\kappa_{d}$ have about the same magnitude. This is because the undistorted distribution $u\left(x, \mathbf{b}_{\perp}\right)$ is twice as large as $d\left(x, \mathbf{b}_{\perp}\right)$, while $\left|\mathcal{E}_{d}\left(x, \mathbf{b}_{\perp}\right)\right| \approx\left|\mathcal{E}_{d}\left(x, \mathbf{b}_{\perp}\right)\right|$, and therefore adding $\frac{1}{2 M} \frac{\partial}{\partial b_{y}} \mathcal{E}_{q}\left(x, \mathbf{b}_{\perp}\right)$ results in a larger distortion for $d$ quarks than for $u$ quarks. Finally one notes that the resulting quark distribution in this state has a transverse (electric) dipole moment

$$
d_{y}=\frac{2}{3} d_{y}^{u}-\frac{1}{3} d_{y}^{d}
$$

where

$$
\begin{aligned}
d_{y}^{q} & \equiv \int_{-1}^{1} d x \int d^{2} \mathbf{b}_{\perp} b_{y} q_{X}\left(x, \mathbf{b}_{\perp}\right)=\frac{1}{2 M} \int_{-1}^{1} d x \int d^{2} \mathbf{b}_{\perp} \mathcal{E}\left(x, \mathbf{b}_{\perp}\right) \\
& =\int_{-1}^{1} d x E_{q}(x, 0,0)=\frac{\kappa_{q}}{2 M},
\end{aligned}
$$

i.e., using Eq. (45), one finds a rather large numerical value for the transverse electric dipole moment due to this distortion

$$
\mu_{y}^{e l .}=0.17 \text { e fm. }
$$

Of course, one could have also read off the order of magnitude from Figs. 3, 4, but in contrast to the model results presented in the figures, Eq. (48) is a model independent result. Furthermore, although the precise numerical value for the transverse electric dipole moment (49) relies on the approximation $\kappa_{s} \approx 0$, a model independent measure of the transverse distortion (relying only on isospin symmetry) is still given by $d_{y}^{u}-d_{y}^{d}=\frac{\kappa_{u}-\kappa_{d}}{2 M}=\frac{\kappa_{p}-\kappa_{n}}{2 M} \approx 0.38 \mathrm{fm}$. Note also that the opposite signs 
October 23, 2018 11:40 WSPC/Guidelines-IJMPA gpda

Impact Parameter Space Interpretation for Generalized Parton Distributions

$$
u\left(x, \mathbf{b}_{\perp}\right)
$$
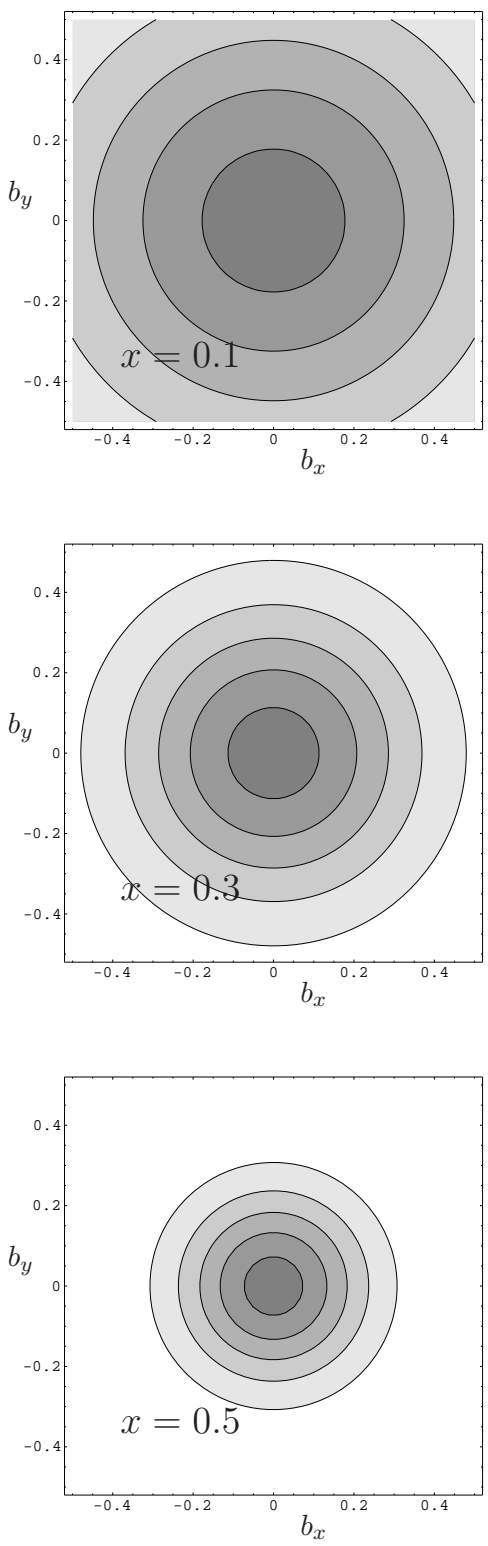

$u_{X}\left(x, \mathbf{b}_{\perp}\right)$
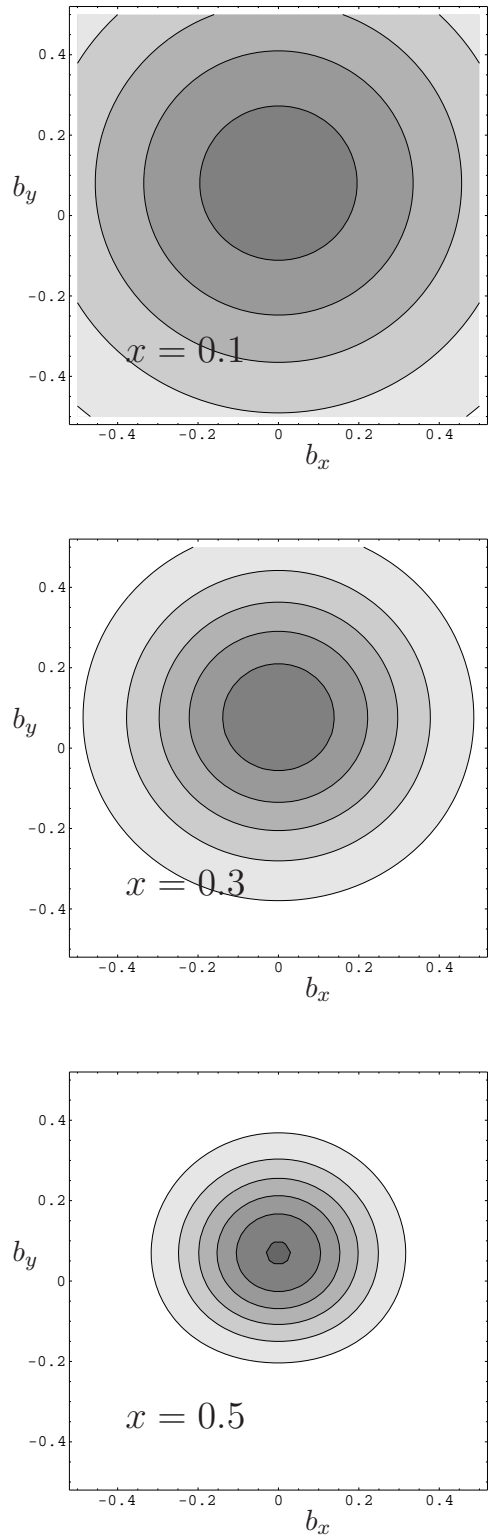

Fig. 3. $u$ quark distribution in the transverse plane for $x=0.1,0.3$, and 0.5 (31). Left column: $u\left(x, \mathbf{b}_{\perp}\right)$, i.e. the $u$ quark distribution for unpolarized protons; right column: $u_{X}\left(x, \mathbf{b}_{\perp}\right)$, i.e. the unpolarized $u$ quark distribution for 'transversely polarized' protons $|X\rangle=|\uparrow\rangle+|\downarrow\rangle$. The distributions are normalized to the central (undistorted) value $u\left(x, \mathbf{0}_{\perp}\right)$. 


$$
d\left(x, \mathbf{b}_{\perp}\right)
$$
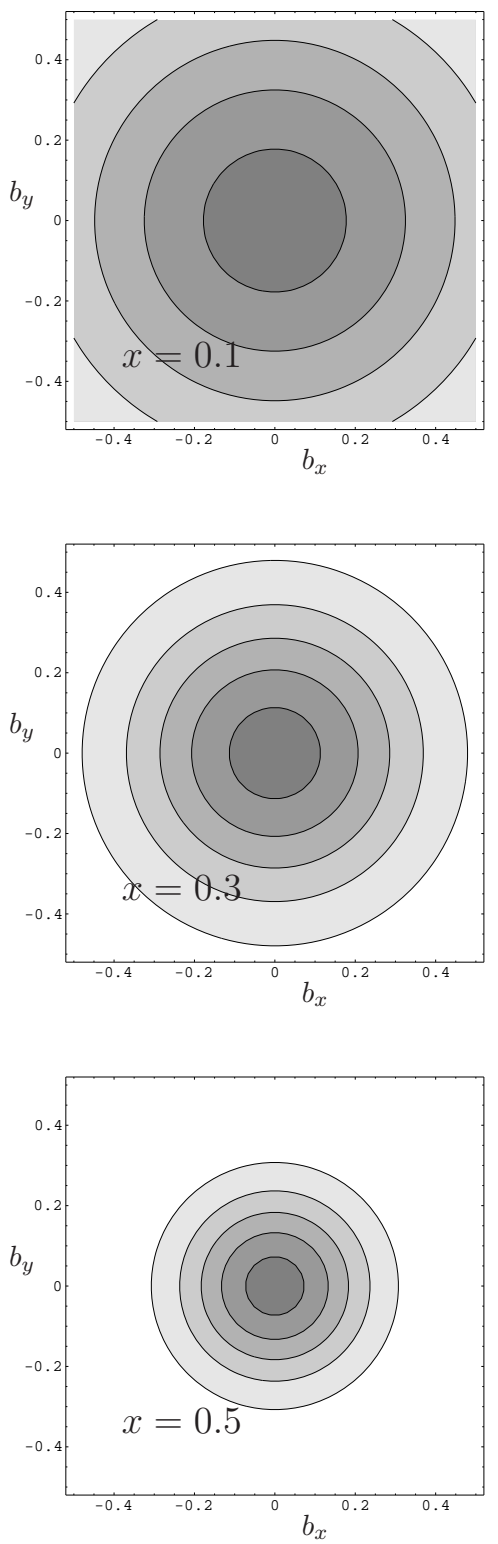

$d_{X}\left(x, \mathbf{b}_{\perp}\right)$
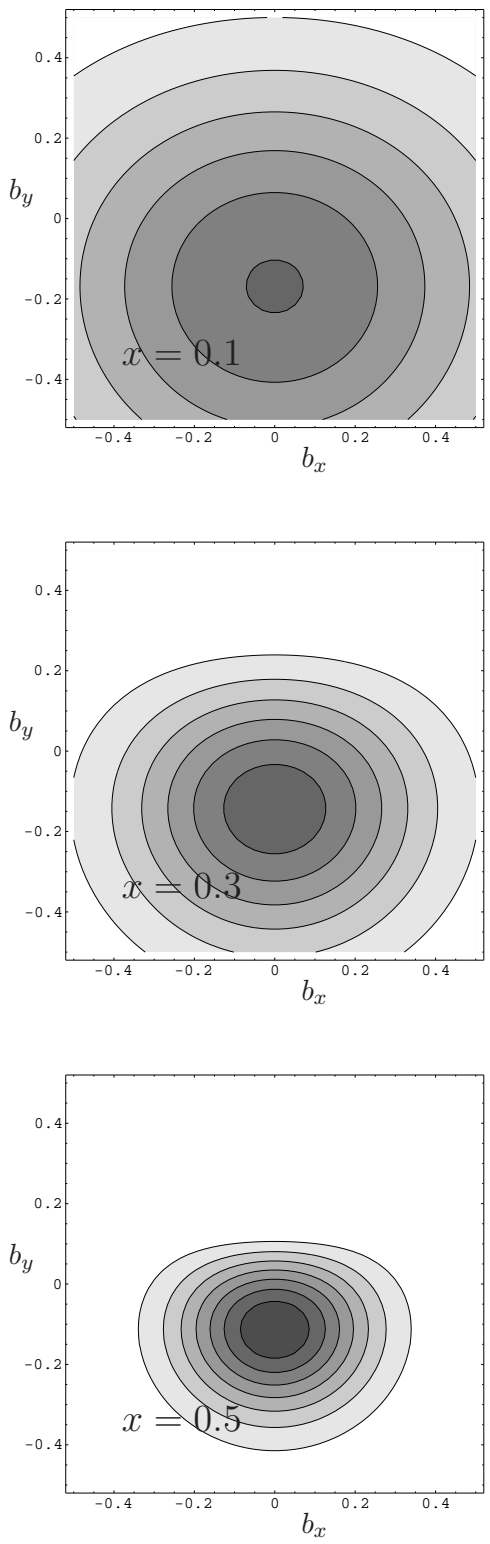

Fig. 4. Same as Fig. 3, but for $d$ quarks.

of $\kappa_{u}$ and $\kappa_{d}$ imply that the distributions of $u$ and $d$ quarks get distorted in opposite directions (Figs. 3 , 团) and therefore they contribute with the same sign (i.e. coherently) to the transverse electric dipole moment. This transverse electric dipole 
moment is perpendicular to both the momentum of the nucleon (along $z$-axis) as well as the polarization (in $x$-direction) and an electric dipole moment pointing in a direction determined by

$$
\vec{\mu}^{e l .} \sim \vec{S} \times \vec{P}
$$

is thus consistent with both parity and time reversal invariance. In fact, one can observe certain similarities with the geometry that arises in single spin assymetries 16 .

\subsection{Implications for Transverse Hyperon Polarization}

The transverse distortion of impact parameter dependent PDFs for states with transverse polarization should have many interesting implications for the production of hadrons with transverse polarization as one can see from the the reactions $P+P \longrightarrow Y+X$ or $P+\bar{P} \longrightarrow Y+X$ with $Y \in\{\Lambda, \Sigma, \Xi\}$ at high energies. We will use plausibility arguments to motivate a rather simple general reaction mechanism and explain the implications for the transverse polarization of produced hyperons.

First we will assume that most hyperons are not produced in central collisions, but instead in more peripheral interactions because at high energy a central collision will most likely yield only an unpolarized background. Secondly, we will assume that the hyperon will be deflected in the direction where the initial proton overlaps with the anti-proton because most reaction mechanisms between $p$ and $\bar{p}$ will give rise to a strong attraction. Finally, we will assume that the $s \bar{s}$ pair is produced in that overlap region and that the produced $s$ quarks form the final state hyperon together with the 'rest' of the initial proton.

These very simple assumptions, together with the transverse distortion of the strange quark cloud in a transversely polarized hyperon will favor hyperons with a specific transverse polarization: Consider first the case where the hyperon has been deflected to the left (Fig. 5), where we look in the direction of the outgoing hyperon. Based on our model assumptions above, the $s$ quark has been produced on the left side of the hyperon, which we assume has a positive strange anomalous magnetic moment $\kappa_{s}^{Y}$ (the case $\kappa_{s}^{Y}<0$ yields the opposite effect). If the final state hyperon is polarized 'up' (w.r.t. the reaction plane) then the $s$ quark distribution in the hyperon is distorted to the right, i.e. away from the reaction zone, while it is distorted to the left (towards the reaction zone) if the polarization is down (Fig. 6). Clearly the second possibility yields a better overlap between the intermediate state and the final state and we would therefore expect a polarization 'down' in this case. The polarization is reversed when the hyperon is deflected to the right (because then the reaction zone is on its right side) and it is also reversed when the sign of $\kappa_{s}^{Y}$ is reversed. These simple considerations lead to the prediction that the polarization direction in this reaction is determined by

$$
\vec{P}_{Y} \sim-\kappa_{s}^{Y} \vec{p}_{P} \times \vec{p}_{Y}
$$


20 Matthias Burkardt

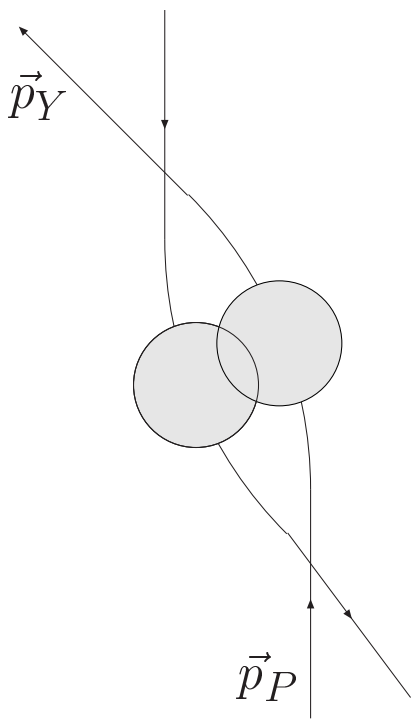

Fig. 5. $\quad P+P(\bar{P}) \longrightarrow Y+\bar{Y}$ where the incoming $P$ (from bottom) is deflected to the left during the reaction. The $s \bar{s}$ pair is assumed to be produced roughly in the overlap region, i.e. on the left 'side' of the $Y$.

a)

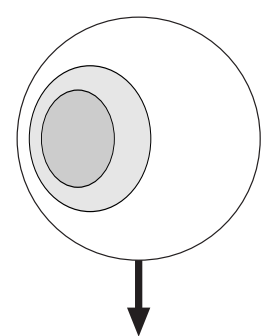

b)

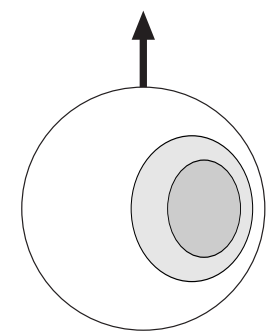

Fig. 6. Schematic view of the transverse distortion of the $s$ quark distribution (in grayscale) in the transverse plane for a transversely polarized hyperon with $\kappa_{s}^{Y}>0$. The view is (from the rest frame) into the direction of motion (i.e. momentum into plane) for a hyperon that moves with a large momentum. In the case of spin down (a), the $s$-quarks get distorted towards the left, while the distortion is to the right for the case of spin up (b).

Before we can compare this remarkably simple prediction to experimental data, we 
need to determine $\kappa_{s}^{Y}$ for various hyperons. Using $S U(3)$ flavor symmetry, one finds

$$
\begin{aligned}
& \kappa_{s}^{\Lambda}=\kappa^{p}+\kappa_{s}^{p}=1.79+\kappa_{s}^{p} \\
& \kappa_{s}^{\Sigma}=\kappa^{p}+2 \kappa^{n}+\kappa_{s}^{p}=-2.03+\kappa_{s}^{p} \\
& \kappa_{s}^{\Xi}=2 \kappa^{p}+\kappa^{n}+\kappa_{s}^{p}=1.67+\kappa_{s}^{p} .
\end{aligned}
$$

Although the exact value for the strange magnetic moment of the nucleon is not known, it is unlikely to be on the same order as $\kappa^{p}$ or $\kappa^{n}$, i.e. Eq. (52) tells us that the strange anomalous magnetic moment of the $\Lambda$ and the $\Xi$ hyperon is positive, while it is negative for the $\Sigma$, yielding for the polarizations

$$
\begin{aligned}
& \vec{P}_{\Lambda} \sim-\vec{p}_{P} \times \vec{p}_{Y} \\
& \vec{P}_{\Sigma} \sim+\vec{p}_{P} \times \vec{p}_{Y} \\
& \vec{P}_{\Xi} \sim-\vec{p}_{P} \times \vec{p}_{Y}
\end{aligned}
$$

which agrees with the experimentally observed pattern of polarizations ( $P$ w.r.t. $\left.\vec{p}_{P} \times \vec{P}_{Y}\right)$

$$
0<P_{\Sigma^{0}} \approx P_{\Sigma^{-}} \approx P_{\Sigma^{+}} \approx-P_{\Lambda} \approx-P_{\Xi^{0}} \approx-P_{\Xi^{-}}
$$

in hyperon production reactions.]

Although we illustrated the effect here in the example of $P+P(\bar{P}) \longrightarrow Y+X$, it should be clear that the effect should also apply to many other hyperon (as well as other hadron) production reactions, but a complete discussion of this subject would go beyond the scope of this section which should serve only as a simple illustration of the striking consequences that the transverse distortion of the parton distribution in transversely polarized may have.

\section{Polarized Impact Parameter Dependent Parton Distributions}

For a longitudinally polarized nucleon, i.e. when $\lambda=\lambda^{\prime}$, the distribution of unpolarized quarks is of course independent of $\lambda$, i.e.

$$
q\left(x, \mathbf{b}_{\perp}\right) \equiv\left\langle p^{+}, \mathbf{R}_{\perp}=0, \lambda\left|\hat{O}_{q}\left(x, \mathbf{b}_{\perp}\right)\right| p^{+}, \mathbf{R}_{\perp}=0, \lambda\right\rangle,
$$

regardless of $\lambda$ (this follows from $\mathrm{PT}$ invariance). In this section we are more interested in impact parameter dependent polarized quark distributions, which we define as

$$
\Delta q\left(x, \mathbf{b}_{\perp}\right) \equiv\left\langle p^{+}, \mathbf{R}_{\perp}=0, \uparrow\left|\hat{O}_{5}\left(x, \mathbf{b}_{\perp}\right)\right| p^{+}, \mathbf{R}_{\perp}=0, \uparrow\right\rangle,
$$

where

$$
\hat{O}_{5}\left(x, \mathbf{b}_{\perp}\right) \equiv \int \frac{d x^{-}}{4 \pi} \bar{q}\left(-\frac{x^{-}}{2}, \mathbf{b}_{\perp}\right) \gamma^{+} \gamma_{5} q\left(\frac{x^{-}}{2}, \mathbf{b}_{\perp}\right) e^{i x p^{+} x^{-}}
$$

\footnotetext{
${ }^{1}$ For a recent discussion of polarization in such reactions, see Ref. 18 and references therein.
} 
An equivalent definition with $\lambda=-\frac{1}{2}$ would involve a minus sign on the r.h.s. of Eq. (56). The physical significance of $\Delta q\left(x, \mathbf{b}_{\perp}\right)$ becomes apparent after we repeat the same steps as in Eqs. (15 16), which yields after some straightforward algebra

$$
\Delta q\left(x, \mathbf{b}_{\perp}\right)=\int \frac{d^{2} \boldsymbol{\Delta}_{\perp}}{(2 \pi)^{2}} \tilde{H}\left(x,-\boldsymbol{\Delta}_{\perp}^{2}\right) e^{-i \mathbf{b}_{\perp} \cdot \boldsymbol{\Delta}_{\perp}},
$$

i.e. by Fourier transform one can relate the impact parameter dependent polarized quark distribution $\Delta q\left(x, \mathbf{b}_{\perp}\right)$ to the polarized GPD $\tilde{H}\left(x,-\boldsymbol{\Delta}_{\perp}^{2}\right)$.

The impact parameter dependent polarized quark distribution also satisfies

$$
\int d^{2} \mathbf{b}_{\perp} \Delta q\left(x, \mathbf{b}_{\perp}\right)=\Delta q(x)
$$

as one might have expected. The probabilistic interpretation of polarized impact parameter dependent quark distributions is rather similar to the one of regular polarized quark distribution: For this purpose, let us first define impact parameter dependent parton distribution for parallel and anti-parallel helicities $(x>0)$,

$$
\begin{aligned}
& q_{\uparrow}\left(x, \mathbf{b}_{\perp}\right) \equiv\left\langle p^{+}, \mathbf{R}_{\perp}=0, \uparrow\left|\hat{O}_{\uparrow}\left(x, \mathbf{b}_{\perp}\right)\right| p^{+}, \mathbf{R}_{\perp}=0, \uparrow\right\rangle, \\
& q_{\downarrow}\left(x, \mathbf{b}_{\perp}\right) \equiv\left\langle p^{+}, \mathbf{R}_{\perp}=0, \uparrow\left|\hat{O}_{\downarrow}\left(x, \mathbf{b}_{\perp}\right)\right| p^{+}, \mathbf{R}_{\perp}=0, \uparrow\right\rangle,
\end{aligned}
$$

with $\hat{O}_{\uparrow \downarrow}\left(x, \mathbf{b}_{\perp}\right)=\hat{O}\left(x, \mathbf{b}_{\perp}\right) \pm \hat{O}_{5}\left(x, \mathbf{b}_{\perp}\right)$. They provide a decomposition of the unpolarized and polarized impact parameter dependent PDF into helicity components, i.e.

$$
\begin{aligned}
q\left(x, \mathbf{b}_{\perp}\right) & =q_{\uparrow}\left(x, \mathbf{b}_{\perp}\right)+q_{\downarrow}\left(x, \mathbf{b}_{\perp}\right) \quad \text { for } x>0 \\
\Delta q\left(x, \mathbf{b}_{\perp}\right) & =q_{\uparrow}\left(x, \mathbf{b}_{\perp}\right)-q_{\downarrow}\left(x, \mathbf{b}_{\perp}\right)
\end{aligned}
$$

and satisfy

$$
\begin{aligned}
& \int d^{2} \mathbf{b}_{\perp} q_{\uparrow}\left(x, \mathbf{b}_{\perp}\right)=q_{\uparrow}(x) \\
& \int d^{2} \mathbf{b}_{\perp} q_{\downarrow}\left(x, \mathbf{b}_{\perp}\right)=q_{\downarrow}(x)
\end{aligned}
$$

Furthermore, like their forward counterparts, they each have a probabilistic interpretation, i.e. $q_{\uparrow}\left(x, \mathbf{b}_{\perp}\right) \geq 0$ and $q_{\downarrow}\left(x, \mathbf{b}_{\perp}\right) \geq 0$, which can also be expressed in the form

$$
\left|q\left(x, \mathbf{b}_{\perp}\right)\right| \geq\left|\Delta q\left(x, \mathbf{b}_{\perp}\right)\right| .
$$

Very similar relations can be obtained for antiquarks, where one can define $(x<0)$

$$
\begin{aligned}
\bar{q}\left(x, \mathbf{b}_{\perp}\right) & \equiv-q\left(-x, \mathbf{b}_{\perp}\right) \\
\Delta \bar{q}\left(x, \mathbf{b}_{\perp}\right) & \equiv \Delta q\left(-x, \mathbf{b}_{\perp}\right)
\end{aligned}
$$

which can also be expressed in terms of (positive) helicity dependent anti-quark distributions $\bar{q}_{\uparrow \downarrow}\left(x, \mathbf{b}_{\perp}\right)$

$$
\begin{aligned}
\bar{q}\left(x, \mathbf{b}_{\perp}\right) & =\bar{q}_{\uparrow}\left(x, \mathbf{b}_{\perp}\right)+\bar{q}_{\downarrow}\left(x, \mathbf{b}_{\perp}\right) \\
\Delta \bar{q}\left(x, \mathbf{b}_{\perp}\right) & =\bar{q}_{\uparrow}\left(x, \mathbf{b}_{\perp}\right)-\bar{q}_{\downarrow}\left(x, \mathbf{b}_{\perp}\right) .
\end{aligned}
$$




\section{Polarized distributions with nucleon helicity flip}

In Sec. 3 we elucidated the physics of the GPD $E_{q}$ by considering the (unpolarized) quark distribution $q_{X}\left(x, \mathbf{b}_{\perp}\right)$ in a target with $\perp$ polarization, and found that $q_{X}\left(x, \mathbf{b}_{\perp}\right)=q\left(x, \mathbf{b}_{\perp}\right)-\frac{1}{2 M} \frac{\partial}{\partial b_{y}} \mathcal{E}_{q}\left(x, \mathbf{b}_{\perp}\right)$, i.e. the unpolarized quark distribution gets distorted when the polarization is not in the longitudinal direction. Naively one would expect that something similar happens for polarized parton distributions in the transverse plane. However, as one can read off from Eq. (4), there is no contribution to the transition amplitude from $\tilde{E}_{q}$ when $\Delta^{+}=0$. As a result, one finds that

$$
\left\langle p^{+}, \mathbf{R}_{\perp}=\mathbf{0}_{\perp}, \lambda\left|\hat{O}_{5, q}\left(x, \mathbf{b}_{\perp}\right)\right| p^{+}, \mathbf{R}_{\perp}=\mathbf{0}_{\perp}, \lambda^{\prime}\right\rangle=\delta_{\lambda \lambda^{\prime}} \Delta q\left(x, \mathbf{b}_{\perp}\right),
$$

without any contribution from $\tilde{E}_{q}$. The physical interpretation of $\tilde{E}_{q}$ must therefore be very different from the interpretation of $E_{q}$. In particular, one should not expect a simple probabilistic interpretation.

When the nucleon is not in a helicity eigenstate but rather a superposition of helicity eigenstates, e.g.

$$
\left|p^{+}, \mathbf{R}_{\perp}=\mathbf{0}_{\perp}, \phi\right\rangle \equiv \cos \phi\left|p^{+}, \mathbf{R}_{\perp}=\mathbf{0}_{\perp}, \uparrow\right\rangle+\sin \phi\left|p^{+}, \mathbf{R}_{\perp}=\mathbf{0}_{\perp}, \downarrow\right\rangle
$$

such that the mean helicity is $h=\cos 2 \phi$ and the mean transverse spin is $S_{x}=$ $\sin 2 \phi$, then the polarized quark distribution $\Delta q_{\phi}\left(x, \mathbf{b}_{\perp}\right)$ in the transverse plane for such a state is obtained as a simple rescalation from the helicity eigenstates, i.e.

$$
\Delta q_{\phi}\left(x, \mathbf{b}_{\perp}\right) \equiv\left\langle p^{+}, \mathbf{R}_{\perp}=\mathbf{0}_{\perp}, \phi\left|\hat{O}_{5}\left(x, \mathbf{b}_{\perp}\right)\right| p^{+}, \mathbf{R}_{\perp}=\mathbf{0}_{\perp}, \phi\right\rangle=h \Delta q\left(x, \mathbf{b}_{\perp}\right) .
$$

This is in contrast to the result for unpolarized quark distributions in the same state

$$
q_{\phi}\left(x, \mathbf{b}_{\perp}\right)=q\left(x, \mathbf{b}_{\perp}\right)-\frac{S_{x}}{2 M} \frac{\partial}{\partial b_{y}} \mathcal{E}_{q}\left(x, \mathbf{b}_{\perp}\right),
$$

One can use these results to derive further positivity constraints. For this purpose, consider the distribution of quarks $(x>0)$

$$
\begin{aligned}
q_{\uparrow, \phi}\left(x, \mathbf{b}_{\perp}\right) & \equiv\left\langle p^{+}, \mathbf{R}_{\perp}=\mathbf{0}_{\perp}, \phi\left|\hat{O}_{\uparrow}\left(x, \mathbf{b}_{\perp}\right)\right| p^{+}, \mathbf{R}_{\perp}=\mathbf{0}_{\perp}, \phi\right\rangle \\
& =q_{\phi}\left(x, \mathbf{b}_{\perp}\right)+\Delta q_{\phi}\left(x, \mathbf{b}_{\perp}\right)
\end{aligned}
$$

with positive helicity in a state with such a mixed helicity. Combining Eq. (70) with (69) one thus obtains

$$
q_{\uparrow, \phi}\left(x, \mathbf{b}_{\perp}\right)=q\left(x, \mathbf{b}_{\perp}\right)-\frac{S_{x}}{2 M} \frac{\partial}{\partial b_{y}} \mathcal{E}_{q}\left(x, \mathbf{b}_{\perp}\right)+h \Delta q\left(x, \mathbf{b}_{\perp}\right) .
$$

Following arguments very similar to the ones in Section 2, one can easily convince oneself that $q_{\uparrow, \phi}\left(x, \mathbf{b}_{\perp}\right)$ should be positive (for $x>0$ ). Since this should hold for arbitrary $\phi$, one easily obtains

$$
\left|q\left(x, \mathbf{b}_{\perp}\right)\right|^{2} \geq\left|\Delta q\left(x, \mathbf{b}_{\perp}\right)\right|^{2}+\left|\frac{1}{2 M} \frac{\partial}{\partial b_{y}} \mathcal{E}_{q}\left(x, \mathbf{b}_{\perp}\right)\right|^{2},
$$


which is stronger than Eqs. (64) and 42. Note that Eq. (73) can also be expressed in the form

$$
\sqrt{\left|q_{\uparrow}\left(x, \mathbf{b}_{\perp}\right) q_{\downarrow}\left(x, \mathbf{b}_{\perp}\right)\right|} \geq \frac{1}{4 M}\left|\nabla_{\mathbf{b}_{\perp}} \mathcal{E}_{q}\left(x, \mathbf{b}_{\perp}\right)\right| .
$$

Using the known asymptotic behavior of $q_{\uparrow}$ and $q_{\downarrow}$ near $x \rightarrow 1$ 17, one can use the latter inequality to place an upper bound on the asymptotic behavior of $\nabla_{\mathbf{b}_{\perp}} \mathcal{E}_{q} \sim$ $(1-x)^{4}$.

\section{Nonzero Skewedness}

Deeply virtual Compton scattering experiments always probe $\xi \neq 0$. Even though polynomiality conditions on the possible $\xi$-dependence 1 should facilitate the extrapolation of GPDs extracted from DVCS experiments to $\xi=0$, it would nevertheless be very desirable to develop a better physical understanding of GPDs at $\xi \neq 0$. In order to better appreciate the difficulties in developing such an interpretation, let us start out by summarizing the problem from an abstract point of view. In general, GPDs have the physical interpretation of a transition amplitude. A necessary condition for a density interpretation is that the initial and final state are the same. This is the case for the usual PDFs but it is not for GPDs, since the latter are distinguished from PDFs due to the fact that $p^{\prime} \neq p$. In the case $\xi=0$, i.e. when $\Delta^{+} \equiv p^{+\prime}-p^{+}$, a Fourier transform of GPDs w.r.t. $\boldsymbol{\Delta}_{\perp} \equiv \mathbf{p}_{\perp}^{\prime}-\mathbf{p}_{\perp}$ diagonalizes the transition amplitude and one can have a density interpretation.

In the case $\Delta^{+} \neq 0$, a Fourier transform w.r.t. $\boldsymbol{\Delta}_{\perp}$ is obviously not enough to render the initial and final state the same, because even after Fourier transforming transverse momenta, the longitudinal momenta are still sharp - and different in the initial and final states. One might be tempted to try using yet another Fourier transform w.r.t. the longitudinal momentum of the target, in order to completely diagonalize GPDs. However, this cannot make sense because the $x$-variable in GPDs already measures the longitudinal momentum of the active quark and Heisenberg's uncertainly relations therefore prohibit the simultaneous measurement of the longitudinal position of the active quark. Even if one would be willing to give up on a precise measurement of $x$, the limitations due to the Heisenberg inequality would severely restrict the longitudinal position space interpretation of GPDs. One can get a rough estimate for the quantitative limitations of the resulting resolution by reminding oneself that the Compton wavelength of a proton is about $1 / 3$ the size of the proton. Therefore even if one measures the momentum of a parton only with an accuracy that is about $1 / 3$ of the nucleons momentum, the resulting position space uncertainty would be of about the same scale as the size of the proton. Therefore, even though one can formally proceed and develop a semiclassical mathematical formalism to extract a parton distribution in 3-dimensional position space $q\left(x, b^{-}, \mathbf{b}_{\perp}\right)$ from GPDs, the limitations due to the uncertainty principle would render this kind of distribution meaningless for a proton target. 
In Refs. 10 a mixed representation (longitudinal momentum/transverse position) was used to simplify the physical interpretation of GPDs with $\xi=0$ as a density. Even though we argued above that such a basis does not lead to a probabilistic interpretation for $\xi \neq 0$, it may nevertheless be useful in simplifying the physical interpretation of GPDs with $\xi \neq 0$, since such a basis may at least partially diagonalize the degrees of freedom.

For this purpose, let us consider a basis of transversely localized basis states $\theta$ that are translated away from the origin

$$
\left|p^{+}, \mathbf{R}_{\perp}=\mathbf{b}_{\perp}, \lambda\right\rangle \equiv \mathcal{N} \int d^{2} \mathbf{p}_{\perp} e^{-i \mathbf{p}_{\perp} \cdot \mathbf{b}_{\perp}}\left|p^{+}, \mathbf{p}_{\perp}, \lambda\right\rangle .
$$

In a recent paper, Diehl pointed out that the Fourier transform of $H_{q}\left(x, \xi,-\Delta_{\perp}^{2}\right)$ can be related in a simple manner to matrix elements of the light-cone correlator between such basis states 9

$$
\begin{aligned}
\int \frac{d^{2} \mathbf{D}_{\perp}}{(2 \pi)^{2}} e^{-i \mathbf{D}_{\perp} \cdot \mathbf{b}_{\perp}} H_{q}\left(x, \xi, \boldsymbol{\Delta}_{\perp}\right) & = \\
& \frac{1+\xi^{2}}{\left(1-\xi^{2}\right)^{5 / 2}}\left\langle p^{\prime+},-\frac{\xi \mathbf{b}_{\perp}}{1-\xi}, \lambda\left|\mathcal{O}_{q}\left(x, \mathbf{b}_{\perp}\right)\right| p^{+}, \frac{\xi \mathbf{b}_{\perp}}{1+\xi}, \lambda\right\rangle,
\end{aligned}
$$

where $\mathbf{D}_{\perp}\left(1-\xi^{2}\right)=\boldsymbol{\Delta}_{\perp}$. Switching to an impact parameter representation yields a transition amplitude that is still off diagonal in $p^{+}$, which is not a surprise. However, on top of that Eq. (76) is also off-diagonal in the impact parameter coordinate, which may be surprising at first since the transition amplitude in Eq. (3) neither changes the transverse position of the spectators (which is trivial) nor does it change the transverse position of the active quark (since $\hat{O}_{q}\left(x, \mathbf{b}_{\perp}\right)$ is diagonal in transverse position). Nevertheless, the transverse center of momentum changes because the $p^{+}$momentum of the active quark changes. As a result, the transverse center of momentum changes and therefore the distance of the active quark to the transverse center of momentum changes. In a sense the situation is comparable to the situation of a decay in a nonrelativistic system when the mass of the active quark changes and therefore the position of the center of mass changes, even if the positions of the individual partons remain the same in the transition process.

However, since the position of the transverse center of mass of the spectators does not change in the transition, it may be useful to switch to a basis, where the transverse position of the active quark is measured w.r.t. the center of momentum of the spectators, rather than the center of momentum of the whole hadron, because the transition amplitude would be diagonal in that basis. Of course, it would still be off-diagonal in the $p^{+}$momentum.

\section{Summary}

Generalized parton distributions provide a light-cone momentum decomposition for form factors in the sense that they tell us how much quarks with a given momentum fraction $x$ contribute to the form factor. 
We considered a (proton) state with a sharp $p^{+}$momentum, which is localized in the $\perp$ direction in the sense that its transverse center of momentum $\mathbf{R}_{\perp} \equiv \sum_{i} x_{i} \mathbf{r}_{\perp, i}$ is at the origin. Such a definition makes sense because there is a Galilean subgroup of transverse boosts in the infinite momentum frame and as a result one can separate the overall $\perp$ momentum from the internal dynamics. This is reminiscent of nonrelativistic dynamics, where one can work in the center of mass frame and where localizing the state by means of a wave packet corresponds to working in a frame where the center of mass is at the origin.

For such a state, we defined the notion of impact parameter dependent parton distribution functions $q\left(x, \mathbf{b}_{\perp}\right)$, where $\mathbf{b}_{\perp}$ is measured relative to the transverse center of momentum $\mathbf{R}_{\perp}$. The significance of these impact parameter dependent PDFs $q\left(x, \mathbf{b}_{\perp}\right)$ is that they are the Fourier transform of the $\xi=0$ GPD $H_{q}\left(x, 0,-\boldsymbol{\Delta}_{\perp}^{2}\right)$. Moreover, $q\left(x, \mathbf{b}_{\perp}\right)$ satisfies a positivity constraint for $x>0$ and a negativity constraint for $x<0$, i.e. one can interpret $q\left(x, \mathbf{b}_{\perp}\right)$ as a density. $m$ This aspect is very important because the probabilistic interpretation underscores the fact that impact parameter dependent PDFs have a physical significance - above and beyond being the Fourier transforms of GPDs w.r.t. $\boldsymbol{\Delta}_{\perp}$.

Conventional parton distributions contain no information about the spatial distribution of partons. If one knows GPDs for $\xi=0$ one can simultaneously determine (by Fourier transform w.r.t. $\boldsymbol{\Delta}_{\perp}$ ) the longitudinal momentum and transverse position of partons in the target. 9 This is completely new information and should provide us with new insights about the internal structure of hadrons. For example, knowledge of GPDs allows to answer questions like 'how the spatial distribution of partons in the nucleons varies with x' or 'what is the parton distribution at a given distance from the center of momentum'.

One of the remarkable results is that there are no relativistic corrections to this interpretation. Formally, this is due to the Galilean subgroup of transverse boosts in the infinite momentum frame. The only limitation in transverse resolution is due to the scale $\frac{1}{Q}$ with which one probes the target. The fact that there are no relativistic corrections to the transverse position space interpretation of GPDs in the IMF implies as a corollary that an interpretation of the Fourier transform of form factors as transverse position space distributions in the IMF either.

$H_{q}\left(x, 0,-\boldsymbol{\Delta}_{\perp}^{2}\right)$ and $\tilde{H}_{q}\left(x, 0,-\boldsymbol{\Delta}_{\perp}^{\mathbf{2}}\right)$ have the most simple physical interpretation as Fourier transforms of unpolarized and polarized impact parameter dependent PDFs respectively for longitudinally polarized nucleons. When the target is polarized in the transverse direction, even the unpolarized quark distribution changes. The resulting distortion of the distribution of quarks in the transverse

${ }^{\mathrm{m}}$ Of course, we have only shown that this probabilistic interpretation holds in the infinite momentum frame (and with light cone gauge), but this is no real drawback since that is anyways the only frame where even forward PDFs have a probabilistic interpretation.

${ }^{n}$ This result is not in contradiction with Heisenberg's uncertainty principle since the information extracted from GPDs is only a simultaneous determination of different components of momentum and position of partons. 
plane is described by the Fourier transform of the nucleon helicity flip distribution $E_{q}\left(x, 0,-\boldsymbol{\Delta}_{\perp}^{2}\right)$. The magnitude of the resulting transverse flavor dipole moment can be related to the anomalous magnetic moment for that flavor in a model independent way. For $\tilde{E}_{q}\left(x, 0,-\boldsymbol{\Delta}_{\perp}^{\mathbf{2}}\right)$ we were unable to find a simple density interpretation, since it does not contribute to matrix elements with purely transverse momentum transfer.

The transverse distortion of the impact parameter dependent PDFs for transversely polarized targets should have important consequences for reactions involving quark production in peripheral scattering as we illustrated in the context of transverse polarization of hyperons.

\section{Acknowledgments}

This work was supported by a grant from DOE (FG03-95ER40965). It is a pleasure to thank Bob Jaffe, Xiangdong Ji, and Andrei Belitsky for very useful discussions.

\section{Appendix A. Galilean Subgroup of Transverse Boosts}

Boost transformations in nonrelativistic quantum mechanics (NRQM)

$$
\begin{aligned}
\vec{x}^{\prime} & =\vec{x}+\vec{v} t \\
t^{\prime} & =t
\end{aligned}
$$

are purely kinematic because they leave the quantization surface $t=0$ invariant. This property has many important consequences. For example, wavefunctions for a many body system in the rest frame and in a boosted frame are related by a simple shift of (momentum) variables, e.g.

$$
\Psi_{\vec{v}}\left(\vec{p}_{1}, \vec{p}_{2}, \vec{p}_{3}\right)=\Psi_{\overrightarrow{0}}\left(\vec{p}_{1}-m_{1} \vec{v}, \vec{p}_{2}-m_{2} \vec{v}, \vec{p}_{3}-m_{3} \vec{v}\right) .
$$

Furthermore, if the Hamiltonian is translationally invariant, the dynamics of the center of mass

$$
\vec{R}=\sum_{i} x_{i} \vec{r}_{i}
$$

with $x_{i}=m_{i} / M$ and $M=\sum_{i} m_{i}$, separates from the intrinsic variables, making it possible to work in the center of mass frame.

One of the features that normally complicates the description of relativistic bound states is that equal time hyperplanes are not invariant under relativistic boosts

$$
\begin{aligned}
\mathbf{x}_{\perp}^{\prime} & =\mathbf{x}_{\perp} \\
z^{\prime} & =\gamma(z+v t) \\
t^{\prime} & =\gamma\left(t+\frac{v}{c^{2}} z\right),
\end{aligned}
$$

with $\gamma^{-2}=1-\frac{v^{2}}{c^{2}}$. As a result, boosts are in general a dynamical operation, the generator of boost transformations contains interactions and there exists no 
simple generalization of Eq. A.2 to a relativistic system quantized at equal times. Furthermore, the notion of the center of mass has no useful generalization in such an equal time quantized relativistic framework.

One of the main advantages of the light-cone (or infinite momentum) framework arise because there is a subgroup of kinematical boosts among the generators of the Poincare group 6 . To see this let us start from the usual Poincaré algebra

$$
\begin{aligned}
{\left[P^{\mu}, P^{\nu}\right] } & =0 \\
{\left[M^{\mu \nu}, P^{\rho}\right] } & =i\left(g^{\nu \rho} P^{\mu}-g^{\mu \rho} P^{\nu}\right) \\
{\left[M^{\mu \nu}, M^{\rho \lambda}\right] } & =i\left(g^{\mu \lambda} M^{\nu \rho}+g^{\nu \rho} M^{\mu \lambda}-g^{\mu \rho} M^{\nu \lambda}-g^{\nu \lambda} M^{\mu \rho}\right)
\end{aligned}
$$

where the generators of rotations and boosts are respectively $M_{i j}=\varepsilon_{i j k} J_{k}$ and $M_{i 0}=K_{i}$.

We now introduce transverse 'boost' operators ${ }^{\circ}$

$$
\begin{aligned}
& B_{x}=\frac{1}{\sqrt{2}}\left(K_{x}+J_{y}\right) \\
& B_{y}=\frac{1}{\sqrt{2}}\left(K_{y}-J_{x}\right)
\end{aligned}
$$

¿From the Poincaré algebra (A.5), it follows that these satisfy commutation relations

$$
\begin{aligned}
{\left[J_{3}, B_{k}\right] } & =i \varepsilon_{k l} B_{l} \\
{\left[P_{k}, B_{l}\right] } & =-i \delta_{k l} P^{+} \\
{\left[P^{-}, B_{k}\right] } & =-i P_{k} \\
{\left[P^{+}, B_{k}\right] } & =0
\end{aligned}
$$

with $k, l \in\{x, y\}, \varepsilon_{x y}=-\varepsilon_{y x}=1$, and $\varepsilon_{x x}=\varepsilon_{y y}=0$. 月 Together with the well known commutation relations

$$
\begin{aligned}
{\left[J_{z}, P_{k}\right] } & =i \varepsilon_{k l} P_{l} \\
{\left[P^{-}, P_{k}\right] } & =\left[P^{-}, P^{+}\right]=\left[P^{-}, J_{z}\right]=0 \\
{\left[P^{+}, P_{k}\right] } & =\left[P^{+}, B_{k}\right]=\left[P^{+}, J_{z}\right]=0
\end{aligned}
$$

these are the same commutation relations as the commutation relations among the generators of the Galilei transformations for NRQM in the plane, provided we make the identifications

$$
\begin{aligned}
& P^{-} \longrightarrow \text { Hamiltonian } \\
& \mathbf{P}_{\perp} \longrightarrow \text { momentum in the plane } \\
& P^{+} \longrightarrow \text { mass }
\end{aligned}
$$

${ }^{\circ}$ Strictly speaking the physical meaning of these operators is a combination of boosts and rotations. $\mathrm{p}$ Using these commutation relations, one easily verifies that $e^{i \mathbf{v}_{\perp} \cdot \mathbf{B}_{\perp}} \mathbf{P}_{\perp} e^{-i \mathbf{v}_{\perp} \cdot \mathbf{B}_{\perp}}=\mathbf{P}_{\perp}+P^{+} \mathbf{v}_{\perp}$, which justifies the identification of $\mathbf{B}_{\perp}$ as a transverse boost operator. 


$$
\begin{aligned}
L_{z} & \longrightarrow \text { rotations around } z \text {-axis } \\
\mathbf{B}_{\perp} & \longrightarrow \text { generator of boosts in the plane. }
\end{aligned}
$$

Because of this isomorphism between transverse boosts in the infinite momentum frame and boosts in the context of NRQM in the plane, many familiar results from NRQM can be directly carried over to relativistic systems in the infinite momentum frame.

In order to construct a localized nucleon state, we start from so called infinite momentum frame helicity states $19\left|p^{+}, \mathbf{p}_{\perp}, \lambda\right\rangle$. They are defined by making use of a Wigner construction, where one starts from a massive particle at rest with spin projection $\lambda$ along the $z$ axis and applies an appropriate boost

$$
\left|p^{+}, \mathbf{p}_{\perp}, \lambda\right\rangle=e^{-i \mathbf{v}_{\perp} \cdot \mathbf{B}_{\perp}}\left|p^{+}, \mathbf{0}_{\perp}, \lambda\right\rangle=e^{-i \mathbf{v}_{\perp} \cdot \mathbf{B}_{\perp}} e^{-i \omega K}\left|M / \sqrt{2}, \mathbf{0}_{\perp}, \lambda\right\rangle,
$$

where $\mathbf{p}_{\perp}=p^{+} \mathbf{v}_{\perp}$ and $e^{\omega}=\sqrt{2} p^{+} / M$. These states are of well defined for any momentum $p^{\mu}$. However, they are most useful to describe particles that move with high velocity in the $z$ direction because, when viewed from the infinite momentum frame these 'light-cone helicity' states also become eigenstates of the ordinary helicity operator. For details we refer to Ref. 19 . For our purposes the most important properties of these states are 19

$$
\begin{aligned}
e^{-i \phi J_{z}}\left|p^{+}, p^{1}, p^{2}, \lambda\right\rangle & =e^{-i \phi \lambda}\left|p^{+}, p^{1} \cos \phi-p^{2} \sin \phi, p^{2} \cos \phi+p^{1} \sin \phi, \lambda\right\rangle \mathrm{A} . \\
e^{-i \mathbf{v}_{\perp} \cdot \mathbf{B}_{\perp}}\left|p^{+}, \mathbf{p}_{\perp}, \lambda\right\rangle & =\left|p^{+}, \mathbf{p}_{\perp}+p^{+} \mathbf{v}_{\perp}, \lambda\right\rangle .
\end{aligned}
$$

¿From these properties it is straightforward to show that these states satisfy

$$
\left|p^{+}, \mathbf{R}_{\perp}=0, \lambda\right\rangle \equiv \mathcal{N} \int d^{2} \mathbf{p}_{\perp}\left|p^{+}, \mathbf{p}_{\perp}, \lambda\right\rangle
$$

are a simultaneous eigenstate of the longitudinal (light-cone) momentum $P^{+}=$ $P^{0}+P^{3}$, the total angular momentum in the $z$ direction $J_{z}$, and the transverse position operator $\mathbf{R}_{\perp}=-\frac{1}{p^{+}} \mathbf{B}_{\perp}$, i.e.

$$
\begin{aligned}
\hat{P}^{+} \mid p^{+}, \mathbf{R}_{\perp} & =0, \lambda\rangle=p^{+}\left|p^{+}, \mathbf{R}_{\perp}=0, \lambda\right\rangle \\
\mathbf{R}_{\perp} \mid p^{+}, \mathbf{R}_{\perp} & =0, \lambda\rangle=0 \\
J_{z} \mid p^{+}, \mathbf{R}_{\perp} & =0, \lambda\rangle=\lambda\left|p^{+}, \mathbf{R}_{\perp}=0, \lambda\right\rangle .
\end{aligned}
$$

In nonrelativistic quantum mechanics, boost transformations shift the momentum of the $i^{\text {th }}$ particle by the amount

$$
\vec{p}_{i} \rightarrow \vec{p}_{i}+\Delta \vec{v} m_{i},
$$

i.e. the generator of boosts is, up to the overall mass of the system, given by the center of mass operator $\vec{B} \equiv-\sum_{i} m_{i} \vec{r}_{i}=-M \vec{R}_{C M}$.

In the infinite momentum frame, $\mathbf{b}_{\perp}$, the generator of $\perp$ boosts, has the physical meaning of the $\perp$ center of momentum times the total momentum $p^{+}$of the system. There are several ways to see this result, which are all worth mentioning since this illustrates the physics of the $\perp$ center of momentum. First this should by obvious 
due to the isomorphism between boosts in nonrelativistic quantum mechanics and $\perp$ boosts in the IMF provided one identifies the nonrelativistic masses $m_{i}$ with the longitudinal momenta $k_{i}^{+}$, i.e. 19

$$
\mathbf{R}_{\mathbf{i}}=-\frac{1}{P^{+}} \mathbf{B}_{i} .
$$

Another way to arrive at the same conclusion is to notice that $\Theta^{++}$, where $\Theta^{\mu \nu}$ is the energy momentum tensor, has the physical meaning of a light-cone momentum density. The field theoretic way of constructing the $\perp$ center of momentum is to weigh the $\perp$ position variable $\mathbf{x}_{\perp}$ with the momentum density $\Theta^{++}$, yielding

$$
\mathbf{R}_{\perp}=\frac{1}{P^{+}} \int d x^{-} d^{2} x_{\perp} \Theta^{++} x_{\perp}=-\frac{1}{P^{+}} \mathbf{B}_{\perp}
$$

which agrees with the result obtained by analogy with nonrelativistic quantum mechanics. Furthermore it shows how the transverse center of momentum operator is related to the generators of the Poincare' group which are of course renormalization group invariant.

The intuitive parton representation for $\mathbf{R}_{\perp}$ in LF gauge is obtained by expressing $\Theta^{++}$in Eq. A.19) in terms of light-cone creation and annihilation operators and noticing that, after integrating over $x^{-}$, only terms that are diagonal in Fock space contribute, yielding

$$
\mathbf{R}_{\perp}=\frac{\sum_{i} k_{i}^{+} r_{\perp, i}}{P_{\text {total }}^{+}}=\sum_{i} x_{i} r_{\perp, i} .
$$

Again, this result should not be surprising, since the longitudinal momentum fractions $x_{i} \equiv k_{i}^{+} / P_{\text {total }}^{+}$play a very similar role as the mass fractions $m_{i} / M_{\text {total }}$ in NRQM.

The reason that $\mathbf{R}_{\perp}$ plays the role of a reference point for impact parameter dependent parton distributions can again be understood by simple analogy with nonrelativistic quantum mechanics. We defined $q\left(x, \mathbf{b}_{\perp}\right)$ starting from the state

$$
\left|p^{+}, \mathbf{R}_{\perp}=\mathbf{0}_{\perp}, \lambda\right\rangle \equiv \mathcal{N} \int d^{2} p_{\perp}\left|p^{+}, \mathbf{p}_{\perp}, \lambda\right\rangle .
$$

¿From the commutation relations it is clear that $\mathbf{b}_{\perp}$ is canonically conjugate to $\mathbf{p}_{\perp}$ and therefore this state satisfies

$$
\mathbf{b}_{\perp}\left|p^{+}, \mathbf{R}_{\perp}=\mathbf{0}_{\perp}, \lambda\right\rangle=0,
$$

just like $\hat{\vec{r}} \int d^{3} p|\vec{p}\rangle=0$ in NRQM. One can see this also directly by noticing that 19

$$
e^{i \mathbf{v}_{\perp} \cdot \mathbf{B}_{\perp}}\left|p^{+}, \mathbf{p}_{\perp}, \lambda\right\rangle=\left|p^{+}, \mathbf{p}_{\perp}+p^{+} \mathbf{v}_{\perp}, \lambda\right\rangle
$$

i.e.

$$
\begin{aligned}
e^{i \mathbf{v}_{\perp} \cdot \mathbf{B}_{\perp}} \int d^{2} p_{\perp}\left|p^{+}, \mathbf{p}_{\perp}, \lambda\right\rangle & =\int d^{2} p_{\perp}\left|p^{+}, \mathbf{p}_{\perp}+p^{+} \mathbf{v}_{\perp}, \lambda\right\rangle \\
& =\int d^{2} p_{\perp}\left|p^{+}, \mathbf{p}_{\perp}, \lambda\right\rangle
\end{aligned}
$$


and therefore $\mathbf{B}_{\perp} \int d^{2} p_{\perp}\left|p^{+}, \mathbf{p}_{\perp}, \lambda\right\rangle=0$.

Because of Eqs. A.19) and (A.22) it is justified to say that $\mathbf{b}_{\perp}$ in $q\left(x, \mathbf{b}_{\perp}\right)$ is measured w.r.t. the transverse center of momentum $\mathbf{R}_{\perp}$.

\section{Appendix B. Overlap Integrals of Light-Cone Wave Functions}

The simple boost properties of LF wave functions have also bee used to construct convenient expression for GPDs in terms of overlap integrals 20. The Galilean invariance implies that $L F$ wavefunctions in a frame with $\Delta_{\perp} \neq 0$ can be obtained from those in the $\Delta_{\perp}=0$ frame by means of a simple shift of variables

$$
\begin{aligned}
p_{i}^{+} \longrightarrow p_{i}^{+^{\prime}}=p_{i}^{+} \\
\mathbf{p}_{\perp, i} \longrightarrow \mathbf{p}_{\perp, i}^{\prime}=\mathbf{p}_{\perp, i}+x_{i} \boldsymbol{\Delta}_{\perp} .
\end{aligned}
$$

For example, for the N-particle Fock space amplitude $\Psi_{\mathbf{P}_{\perp}}^{N}$ for a state with overall momentum $\mathbf{P}_{\perp}$, one finds

$$
\begin{aligned}
\Psi_{\mathbf{P}_{\perp}}^{N}\left(x_{1}, \mathbf{k}_{\perp, 1}, x_{2}, \mathbf{k}_{\perp, 2}, \ldots, x_{N}, \mathbf{k}_{\perp, N}\right)= & \Psi_{\mathbf{0}_{\perp}}^{N}\left(x_{1}, \mathbf{k}_{\perp, 1}-x_{1} \mathbf{P}_{\perp}, x_{2}, \mathbf{k}_{\perp, 2}-x_{2} \mathbf{P}_{\perp}, \ldots\right) \\
& \times \delta\left(\mathbf{P}_{\perp}-\sum_{i=1}^{N} \mathbf{k}_{\perp, i}\right) .
\end{aligned}
$$

Note that the momentum fractions $x_{i} \equiv \frac{p_{i}^{+}}{\sum_{i} p_{i}^{+}}$carried by the $i^{\text {th }}$ parton play a similar role here as the mass fractions in the corresponding nonrelativistic boost formula.

Using this very simple boost property one can express typical overlap integrals that appear within the context of form factors and GPDs in terms of overlap integrals involving internal momenta only (B.3). For purely transverse momentum transfers $(\xi=0)$ these overlap integrals take on a particularly simple form in terms of LC wave functions (Fock space amplitudes) $20 \Psi_{N}\left(x, \mathbf{k}_{\perp}\right)$

$$
H_{q}\left(x,-\boldsymbol{\Delta}_{\perp}^{2}\right)=\sum_{N} \sum_{j} \int[d x]_{N} \int\left[d^{2} \mathbf{k}_{\perp}\right]_{N} \delta\left(x-x_{j}\right) \Psi_{N}^{*}\left(x_{i}, \mathbf{k}_{\perp, i}^{\prime}, \lambda_{i}\right) \Psi_{N}\left(x_{i}, \mathbf{k}_{\perp, i}, \lambda_{i}\right)
$$

where $\mathbf{k}_{\perp, i}^{\prime}=\mathbf{k}_{\perp, i}-x_{i} \boldsymbol{\Delta}_{\perp}$ for $i \neq j$ and $\mathbf{k}_{\perp, j}^{\prime}=\mathbf{k}_{\perp, j}+\left(1-x_{j}\right) \boldsymbol{\Delta}_{\perp}$. The helicities of the partons are labeled by $\lambda_{i}$ (In the following we will only consider amplitudes that are diagonal in the helicities and we will therefore omit the helicity labels in all expressions in order to simplify the notation).

Note that Eq. (B.3) is very similar to the expression for the form factor in the Drell-Yan frame, except that the $x$ of the 'active' quark is not integrated over, and it is exact if one knows the $\Psi_{N}$ for all Fock components. The overlap integrals in terms of momentum space wavefunctions in Eq. (B.3) are very much reminiscent of similar expressions for form factors of nonrelativistic systems. In order to explore this 
analogy further, we consider Fock space amplitudes $\tilde{\Psi}_{\mathbf{P}_{\perp}}^{N}$ in a 'mixed' (longitudinal momentum and transverse position space) representation

$$
\tilde{\Psi}_{\mathbf{P}_{\perp}}^{N}\left(x_{i}, \mathbf{r}_{\perp, i}\right) \equiv \int\left[d^{2} \mathbf{k}_{\perp}\right] e^{i \sum_{i} \mathbf{r}_{\perp, i} \mathbf{k}_{\perp, i}} \Psi_{\mathbf{P}_{\perp}}^{N}\left(x_{i}, \mathbf{k}_{\perp, i}\right) .
$$

The fact that Fock space amplitudes depend only on 'relative' individual momenta $\hat{\mathbf{k}}_{\perp, i} \equiv \mathbf{k}_{\perp, i}-x_{i} \mathbf{P}_{\perp}$, implies that the position space wavefunction (Fock space amplitude) $\tilde{\Psi}^{N}$ depends on the overall transverse momentum $\mathbf{P}_{\perp}$ only through an overall phase, i.e. it can be expressed in the form

$$
\begin{aligned}
\tilde{\Psi}_{\mathbf{P}_{\perp}}^{N}\left(x_{i}, \mathbf{r}_{\perp, i}\right) & =\int\left[d^{2} \mathbf{k}_{\perp}\right]_{N} e^{i \sum_{i} \mathbf{k}_{\perp, i} \mathbf{r}_{\perp, i}} \Psi_{\mathbf{P}_{\perp}}\left(x_{i}, \mathbf{k}_{\perp, i}\right) \\
& =e^{i \sum_{i} x_{i} \mathbf{P}_{\perp} \mathbf{r}_{\perp, i}} \int\left[d^{2} \hat{\mathbf{k}}_{\perp}\right]_{N} e^{i \hat{\mathbf{k}}_{\perp, i} \mathbf{r}_{\perp, i}} \Psi_{\mathbf{0}_{\perp}}\left(x_{i}, \mathbf{k}_{\perp, i}\right) \\
& =e^{i \mathbf{P}_{\perp} \mathbf{R}_{\perp}} \tilde{\Psi}_{r e l .}^{N}\left(x_{i}, \hat{\mathbf{r}}_{\perp, i}\right),
\end{aligned}
$$

where

$$
\mathbf{R}_{\perp} \equiv \sum_{i} x_{i} \mathbf{r}_{\perp, i}
$$

is the transverse center of momentum and the $\hat{\mathbf{r}}_{i}$ are some 'relative' coordinates, e.g.

$$
\hat{\mathbf{r}}_{\perp, i} \equiv \mathbf{r}_{\perp, i}-\mathbf{R}_{\perp} .
$$

In order to illustrate the physics consequences of this decoupling of the overall transverse momentum for $H_{q}(x, t)$, let us consider the specific cases $N=2$ and $N=3$. 9 In a two particle system

$$
H_{q}\left(x,-\Delta_{\perp}^{2}\right)=\int d^{2} \mathbf{k}_{\perp} \psi_{\boldsymbol{\Delta}_{\perp}}^{*}\left(x, \mathbf{k}_{\perp}+\boldsymbol{\Delta}_{\perp}\right) \psi_{\mathbf{0}_{\perp}}\left(x, \mathbf{k}_{\perp}\right),
$$

and using B.5

$$
\begin{gathered}
\psi_{\mathbf{0}_{\perp}}\left(x, \mathbf{k}_{\perp}\right)=\mathcal{N} \int d^{2} \mathbf{r}_{\perp, 1} d^{2} \mathbf{r}_{\perp, 2} e^{-i \mathbf{k}_{\perp}\left(\mathbf{r}_{\perp, 1}-\mathbf{r}_{\perp, 2}\right)} \tilde{\psi}\left(x, \mathbf{r}_{\perp}\right) \\
\psi_{\boldsymbol{\Delta}_{\perp}}\left(x, \mathbf{k}_{\perp}+\boldsymbol{\Delta}_{\perp}\right)=\mathcal{N} e^{i \boldsymbol{\Delta}_{\perp} \mathbf{R}_{\perp} \int d^{2} \mathbf{r}_{\perp, 1} d^{2} \mathbf{r}_{\perp, 2} e^{-i\left(\mathbf{k}_{\perp}+\boldsymbol{\Delta}_{\perp}\right) \mathbf{r}_{\perp, 1}} e^{i \mathbf{k}_{\perp} \mathbf{r}_{\perp, 2}} \tilde{\psi}\left(x, \mathbf{r}_{\perp}\right),}
\end{gathered}
$$

where $\mathcal{N}$ is some normalization constant such that $N^{2} \int d^{2} \mathbf{R}_{\perp}=1$, one straightforwardly finds

$$
H_{q}\left(x,-\boldsymbol{\Delta}^{2}\right)=\int d^{2} \mathbf{r}_{\perp} e^{i\left(\mathbf{r}_{\perp, 1}-\mathbf{R}_{\perp}\right) \cdot \boldsymbol{\Delta}_{\perp}}\left|\tilde{\psi}\left(x, \mathbf{r}_{\perp}\right)\right|^{2} .
$$

Eq. (B.10) makes it clear that $H_{q}\left(x,-\boldsymbol{\Delta}_{\perp}^{2}\right)$ is the Fourier transform of a density. Furthermore, one can easily read off that the relevant transverse position scale is not set by the separation $\mathbf{r}_{\perp} \equiv \mathbf{r}_{\perp, 1}-\mathbf{r}_{\perp, 2}$ between the quark and the antiquark

वWe may consider each Fock component $N$ separately since $H_{q}(x, t)$ is diagonal in Fock space! 
but by the separation of the active quark from the transverse center of momentum (B.6), which in the center of momentum frame coincides with the impact parameter of the active quark

$$
\mathbf{b}_{\perp, 1} \equiv \mathbf{r}_{\perp, 1}-\mathbf{R}_{\perp}=(1-x) \mathbf{r}_{\perp} .
$$

Starting from the light-cone wave functions, we have thus verified the interpretation of the generalized parton distribution $H_{q}\left(x,-\boldsymbol{\Delta}^{2}\right)$ as the Fourier transform of the probability distribution for the active quark with respect to its impact parameter in the case of a two particle system.

For a three particle system, we start from

$$
H_{q}\left(x,-\boldsymbol{\Delta}_{\perp}^{2}\right)=\int d^{2} \mathbf{k}_{\perp, 1} \int d^{2} \mathbf{k}_{\perp, 2} \int d y \psi_{\boldsymbol{\Delta}_{\perp}}^{*}\left(x, \mathbf{k}_{\perp, 1}+\boldsymbol{\Delta}_{\perp}, y, \mathbf{k}_{\perp, 2}\right) \psi_{\mathbf{0}_{\perp}}\left(x, \mathbf{k}_{\perp, 1}, y, \mathbf{k}_{\perp, 2}\right),
$$

and use $(B .5)$

$$
\begin{aligned}
& \psi_{\mathbf{0}_{\perp}}\left(x, \mathbf{k}_{\perp, 1}, y, \mathbf{k}_{\perp, 2}\right)= \\
& \mathcal{N} \int d^{2} \mathbf{r}_{\perp, 1} d^{2} \mathbf{r}_{\perp, 2} d^{2} \mathbf{r}_{\perp, 3} e^{-i \mathbf{k}_{\perp, 1}\left(\mathbf{r}_{\perp, 1}-\mathbf{r}_{\perp, 3}\right)} e^{-i \mathbf{k}_{\perp, 2}\left(\mathbf{r}_{\perp, 2}-\mathbf{r}_{\perp, 3}\right)} \tilde{\psi}\left(x, \hat{\mathbf{r}}_{\perp, 1}, y, \hat{\mathbf{r}}_{\perp, 2}\right) \\
& \psi_{\boldsymbol{\Delta}_{\perp}}\left(x, \mathbf{k}_{\perp, 1}+\boldsymbol{\Delta}_{\perp}, y, \mathbf{k}_{\perp, 2}\right)= \\
& \mathcal{N} e^{i \boldsymbol{\Delta}_{\perp} \mathbf{R}_{\perp}} \int d^{2} \mathbf{r}_{\perp, 1} d^{2} \mathbf{r}_{\perp, 2} d^{2} \mathbf{r}_{\perp, 3} e^{-i\left(\mathbf{k}_{\perp, 1}+\boldsymbol{\Delta}_{\perp}\right) \mathbf{r}_{\perp, 1}} e^{-i \mathbf{k}_{\perp, 2} \mathbf{r}_{\perp, 2}} e^{i\left(\mathbf{k}_{\perp, 1}+\mathbf{k}_{\perp, 2}\right) \mathbf{r}_{\perp, 3}} \\
& \times \tilde{\psi}\left(x, \hat{\mathbf{r}}_{\perp, 1}, y, \hat{\mathbf{r}}_{\perp, 2}\right),
\end{aligned}
$$

where $\mathcal{N}$ is again some normalization constant. Upon inserting (B.13) into (B.12), one finds after some straightforward algebra

$$
\begin{aligned}
& H_{q}\left(x,-\boldsymbol{\Delta}_{\perp}^{2}\right)=\mathcal{N} e^{i \boldsymbol{\Delta}_{\perp} \mathbf{R}_{\perp}} \int d^{2} \mathbf{r}_{\perp, 1} d^{2} \mathbf{r}_{\perp, 2} d^{2} \mathbf{r}_{\perp, 3} e^{i \boldsymbol{\Delta}_{\perp} \mathbf{r}_{\perp, 1}}\left|\tilde{\psi}\left(x, \hat{\mathbf{r}}_{\perp, 1}, y, \hat{\mathbf{r}}_{\perp, 2}\right)\right|^{2}, \\
& =\int d^{2} \mathbf{r}_{\perp, 1} d^{2} \mathbf{r}_{\perp, 2} e^{i \boldsymbol{\Delta}_{\perp}\left(\mathbf{r}_{\perp, 1}-\mathbf{R}_{\perp}\right)}\left|\tilde{\psi}\left(x, \hat{\mathbf{r}}_{\perp, 1}, y, \hat{\mathbf{r}}_{\perp, 2}\right)\right|^{2},
\end{aligned}
$$

i.e. again we confirm the physical interpretation of $H_{q}\left(x, 0,-\boldsymbol{\Delta}_{\perp}^{2}\right)$ as the Fourier transform of a density.

The generalization of this light-cone wave function based derivation of our general result to arbitrary numbers of partons is straightforward and is left as an exercise for the reader.

\section{References}

1. D. Müller et al., Fortschr. Phys. 42, 101 (1994).

2. X.Ji, J. Phys. G 24, 1181 (1998); A.V. Radyushkin, Phys. Rev. D 56, 5524 (1997); K. Goeke, M.V. Polyakov, and M. Vanderhaeghen, Prog. Part. Nucl. Phys. 47, 401 (2001), A.V. Belitsky and D. Müller, hep-ph/0206306.

3. X. Ji, Phys. Rev. Lett. 78, 610 (1997). 
4. A.V. Radyushkin, Phys. Rev. D 58, 114008 (1998); M. Diehl et al., Phys. Lett. B 460, 204 (1999); Eur. Phys. J. C8, 409 (1999); M. Vanderhaeghen, Eur. Phys. J. A8, 455 (2000); R. Jakob, hep-ph/0010158; P.Kroll, hep-ph/0011280;

5. J.P. Ralston and B. Pire, hep-ph/0110075: J.P. Ralston, R.V. Buniy, and P. Jain, hep-ph/0206063.

6. M. Burkardt, Phys. Rev. D 62, 071503 (2000).

7. J. Kogut and D.E. Soper, Phys. Rev. D 1, 2901 (1970).

8. J.P. Ralston and D. Soper, Nucl. Phys. B152, 109 (1979); J.C. Collins, Nucl. Phys. B396, 161 (1993); P.J. Mulders and R.D. Tangerman, Nucl. Phys. B461 197 (1996); B484, 538 (1997) (E).

9. M. Diehl, hep-ph/0205208.

10. M. Burkardt, proceedings of the workshop on Lepton Scattering, Hadrons and QCD, Eds. W.Melnitchouk et al., Adelaide 2001; hep-ph/0105324.

11. M. Burkardt, Adv. Nucl. Phys. 23, 1 (1996).

12. R.P. Feynman, Photon Hadron Interactions, Reading, Mass., 1972.

13. M. Diehl, Eur. Phys. J. C19, 485 (2001); P.V. Pobylitsa, hep-ph/0201030.

14. S.J. Brodsky et al., Nucl. Phys. B 593, 311 (2001).

15. P.V. Pobylitsa, hep-ph/0204337.

16. J.C. Collins, Nucl. Phys. B 396,161 (1993); D. Boer and P.J. Mulders, Phys. Rev. D 57, 5780 (1998); S.J. Brodsky, D.S. Hwang, and I. Schmidt, hep-ph/0201296.

17. S.J. Brodsky, M. Burkardt, and I. Schmidt, Nucl. Phys. B 441, 197 (1995).

18. S.M. Troshin and N.E. Tyurin, hep-ph/0201267.

19. D.E. Soper, Phys. Rev. D 5, 1956 (1972).

20. M. Diehl et al., Nucl. Phys. B 596, 33 (2001). 